\title{
Ensemble Data Assimilation with the NCEP Global Forecast System
}

\author{
Jeffrey S. Whitaker, Thomas M. Hamill, And Xue Wei \\ NOAA/Earth System Research Laboratory, Boulder, Colorado \\ Yucheng Song ANd Zoltan Toth \\ NCEP/Environmental Modeling Center, Camp Springs, Maryland
}

(Manuscript received 5 September 2006, in final form 30 April 2007)

\begin{abstract}
Real-data experiments with an ensemble data assimilation system using the NCEP Global Forecast System model were performed and compared with the NCEP Global Data Assimilation System (GDAS). All observations in the operational data stream were assimilated for the period 1 January-10 February 2004, except satellite radiances. Because of computational resource limitations, the comparison was done at lower resolution (triangular truncation at wavenumber 62 with 28 levels) than the GDAS real-time NCEP operational runs (triangular truncation at wavenumber 254 with 64 levels). The ensemble data assimilation system outperformed the reduced-resolution version of the NCEP three-dimensional variational data assimilation system (3DVAR), with the biggest improvement in data-sparse regions. Ensemble data assimilation analyses yielded a 24-h improvement in forecast skill in the Southern Hemisphere extratropics relative to the NCEP 3DVAR system (the 48-h forecast from the ensemble data assimilation system was as accurate as the 24-h forecast from the 3DVAR system). Improvements in the data-rich Northern Hemisphere, while still statistically significant, were more modest. It remains to be seen whether the improvements seen in the Southern Hemisphere will be retained when satellite radiances are assimilated. Three different parameterizations of background errors unaccounted for in the data assimilation system (including model error) were tested. Adding scaled random differences between adjacent 6-hourly analyses from the NCEP-NCAR reanalysis to each ensemble member (additive inflation) performed slightly better than the other two methods (multiplicative inflation and relaxation-to-prior).
\end{abstract}

\section{Introduction}

Ensemble-based data assimilation (EDA) methods are emerging as alternatives to four-dimensional variational data assimilation (4DVAR) methods (e.g., Thépaut and Courtier 1991; Courtier et al. 1994; Rabier et al. 2000) for operational atmospheric data assimilation systems. All EDA algorithms are inspired by the Kalman filter (KF), though in EDA the background-error covariances are estimated from an ensemble of shortterm model forecasts instead of propagating the back-

Corresponding author address: Jeffery S. Whitaker, NOAA/ Earth System Research Laboratory, R/PSD1, 325 Broadway, Boulder, CO 80305-3328.

E-mail: jeffrey.s.whitaker@noaa.gov ground-error covariance matrix explicitly with a linear model. Since the KF provides the optimal solution to the data assimilation problem when the error dynamics are linear, and the error covariances are Gaussian and perfectly known, EDA methods also are optimal under the same conditions, as long as the ensemble size is large enough. EDA systems have developed along two primary lines: stochastic filters, which use random number realizations to simulate observation error (e.g., Burgers et al. 1998; Houtekamer and Mitchell 1998), and deterministic filters (e.g., Tippett et al. 2003; Whitaker and Hamill 2002; Anderson 2001; Bishop et al. 2001; Ott et al. 2004), which do not. Comprehensive overviews of ensemble data assimilation techniques can be found in Evensen (2003) and Hamill (2006). EDA methods are potentially attractive alternatives to 4DVAR mainly for three reasons. First, they are very simple to code and maintain, for there is no variational 
minimization involved, and no adjoint of the forecast model is necessary. ${ }^{1}$ Second, they automatically provide an ensemble of states to initialize ensemble forecasts, eliminating the need to run additional algorithms to generate perturbed initial conditions. Third, it is relatively straightforward to treat the effects of model error. In simple models, EDA methods have been shown to perform similarly to 4DVAR, as long as the assimilation window in $4 \mathrm{DVAR}$ is long enough, and better than 4DVAR if the assimilation window is too short (Kalnay et al. 2007).

Although most studies to date have tested EDA systems either in idealized models or under perfect model assumptions, there has been progress recently on testing EDA systems with real-weather prediction models and observations. Whitaker et al. (2004) and Compo et al. (2006) showed that EDA systems are well suited to the problem of historical reanalysis, since the flowdependent background error estimates they provide are especially important when observations are sparse (e.g., Hamill and Snyder 2000; Bouttier 1994). Houtekamer et al. (2005) have implemented an EDA system at the Meteorological Service of Canada (MSC), and their initial implementation was shown to perform similarly to the then-operational system [based on three-dimensional variational data assimilation (3DVAR)]. In this study we have compared the performance of our EDA system with that of a reduced-resolution version of the National Centers for Environmental Prediction (NCEP) operational 3DVAR Global Data Assimilation System (GDAS; Parrish and Derber 1992; Derber and Wu 1998; NCEP Environmental Modeling Center 2004) for the period 1 January-10 February 2004.

The ensemble data assimilation system and experimental design are described in section 2 . The results of the EDA experiments are presented in section 3 and compared with the benchmark run of the NCEP GDAS. Particular attention is paid to the sensitivity of the results to the method for parameterizing model error. In section 3d, our EDA algorithm is compared with a different implementation developed at the University of Maryland called the local ensemble transform Kalman filter (LETKF; Hunt et al. 2007, hereinafter HKS). The results are summarized in the final section and their implications for the further development of ensemble data assimilation are discussed.

\footnotetext{
${ }^{1}$ The adjoint of the forecast model is not strictly necessary for 4DVAR, but is used to iteratively minimize the cost function in all current operational implementations.
}

\section{Experimental design}

\section{a. Observations}

All of the observations used in the NCEP GDAS during the period 1 January 1-10 February 2004, except the satellite radiances, are input into the ensemble data assimilation system. The decision not to include satellite radiances in our initial tests of the EDA was made partially to lessen the computational expense and, thereby, permit a larger number of experiments to be run. Since the effective assimilation of satellite radiances depends crucially on issues related to bias correction, quality control, and radiative transfer, we also felt that withholding radiance observations would simplify a comparison of the methods used to calculate the analysis increment. Since the background-error covariances in the operational NCEP GDAS system were tuned for a higher-resolution forecast model and an observation network that includes satellite radiances, we retuned the background error variances used in the GDAS benchmark run to improve the reducedresolution analysis without satellite radiances (see the appendix for details). It is possible that the GDAS benchmark could be improved with further tuning, but we believe it provides a reasonable baseline for measuring EDA performance.

The calculation of the forward operator $(\mathbf{H})$ was performed by running the NCEP GDAS system once for each ensemble member, saving the values of $\mathbf{H x}^{b}$ (where $\mathbf{x}^{b}$ is the background, or first-guess model forecast) to a file, and exiting the code before the computation of the analysis increment. The observation-error covariances $(\mathbf{R})$ were set to the same values used in the NCEP GDAS.

\section{b. The forecast model and benchmark}

The forecast model used is the forecast model component NCEP Global Forecast System (GFS). We have used a version that was operational in March 2004. The GDAS that was operational at the time (Environmental Modeling Center 2003) uses a first-guess forecast run at a triangular truncation at wavenumber 254 , with 64 sigma levels (T254L64). Computational constraints required us to use a lower resolution for the ensemble data assimilation system (T62L28), with the uppermost level at approximately $3 \mathrm{hPa}$. A digital filter (Lynch and

\footnotetext{
${ }^{2}$ A detailed description of these observations is available online (http://www.emc.ncep.noaa.gov/mmb/data_processing/prepbufr. doc/table_2.htm). There are approximately 250 000-350 000 nonradiance observations at each analysis time, including satellite cloud drift winds.
} 
Huang 1992) with a span of $6 \mathrm{~h}$ centered on the 3-h forecast is performed during the 6-h first-guess forecast, as in the operational GDAS. The digital filter diminishes gravity wave oscillations by temporally filtering the model variables. The performance of the ensemble data assimilation system is evaluated relative to a special run of the NCEP GDAS operational in March 2004 using the same reduced-resolution forecast model and the same reduced set of nonradiance observations, but with the background error variances retuned to account for the reduced resolution of the forecast model and the exclusion of satellite radiances as described in the appendix. We call the analyses generated from this special reduced-resolution run of the GDAS the "NCEPBenchmark," while the operational GDAS analyses are referred to as "NCEP-Operational." The NCEPOperational analyses were run at much higher resolution (T254L64) and included satellite radiances in the assimilation. The quality of the analyses produced by the EDA system are assessed by performing single deterministic forecasts initialized from the ensemblemean EDA analyses and the NCEP-Benchmark analyses, with the same T62L28 version of the NCEP GFS. These forecasts are verified against observations and against the NCEP-Operational analyses.

\section{c. Computing the analysis increment}

Ensemble data assimilation systems are designed to update a forecast ensemble to produce an analysis ensemble with an improved mean and perturbations that sample the analysis-error covariance. Following the notation of Ide et al. (1997), let $\mathbf{x}^{b}$ be an $m$-dimensional background model forecast, let $\mathbf{y}^{o}$ be a $p$-dimensional set of observations, let $\mathbf{H}$ be the operator that converts the model state to the observation space, let $\mathbf{P}^{b}$ be the $m \times m$-dimensional background-error covariance matrix, and let $\mathbf{R}$ be the $p \times p$-dimensional observationerror covariance matrix. The minimum error-variance estimate of the analyzed state $\mathbf{x}^{a}$ is then given by the traditional Kalman filter update equation (Lorenc 1986):

$$
\mathbf{x}^{a}=\mathbf{x}^{b}+\mathbf{K}\left(\mathbf{y}^{o}-\mathbf{H} \mathbf{x}^{b}\right)
$$

where

$$
\mathbf{K}=\mathbf{P}^{b} \mathbf{H}^{\mathrm{T}}\left(\mathbf{H} \mathbf{P}^{b} \mathbf{H}^{\mathrm{T}}+\mathbf{R}\right)^{-1} .
$$

In ensemble data assimilation, $\mathbf{P}^{b} \mathbf{H}^{\mathrm{T}}$ is approximated by using the sample covariance estimated from an ensemble of model forecasts. For the rest of the paper, the symbol $\mathbf{P}^{b}$ is used to denote the sample covariance from an ensemble, and $\mathbf{K}$ is understood to be computed using sample covariances. Expressing the model state vector as an ensemble mean (denoted by an overbar) and a deviation from the mean (denoted by a prime), the update equations for the ensemble square root filter (EnSRF; Whitaker and Hamill 2002) may be written as

$$
\begin{aligned}
\overline{\mathbf{x}}^{a} & =\overline{\mathbf{x}}^{b}+\mathbf{K}\left(\mathbf{y}^{o}-\mathbf{H} \overline{\mathbf{x}}^{b}\right) \quad \text { and } \\
\mathbf{x}^{\prime a} & =\mathbf{x}^{\prime b}-\tilde{\mathbf{K}} \mathbf{H} \mathbf{x}^{\prime b}
\end{aligned}
$$

where $\mathbf{P}^{b} \mathbf{H}^{\mathrm{T}}=\overline{\mathbf{x}^{\prime b}\left(\mathbf{H x}^{\prime b}\right)^{\mathrm{T}}} \equiv(1 / n-1) \sum_{i=1}^{n} \mathbf{x}_{i}^{\prime b}\left(\mathbf{H x}_{i}^{\prime b}\right)^{\mathrm{T}}$, $\mathbf{H P}^{b} \mathbf{H}^{\mathrm{T}}=\overline{\mathbf{H x}^{\prime b}\left(\mathbf{H x}^{\prime b}\right)^{\mathrm{T}}} \equiv(1 / n-1) \sum_{i=1}^{n} \mathbf{H} \mathbf{x}_{i}^{\prime b}\left(\mathbf{H x}_{i}^{\prime b}\right)^{\mathrm{T}}, n$ is the ensemble size ( $=100$ unless otherwise noted), $\mathbf{K}$ is the Kalman gain given by (2), and $\tilde{\mathbf{K}}$ is the gain used to update deviations from the ensemble mean. Note that an overbar used in a covariance estimate implies a factor of $n-1$ instead of $n$ in the denominator, so that the estimate is unbiased. If $\mathbf{R}$ is diagonal, observations may be assimilated serially, one at a time, so that the analysis after assimilation of the $N$ th observation becomes the background estimate for assimilating the $(N+1)$ observation (Gelb et al. 1974). With this simplification, $\tilde{\mathbf{K}}$ may be written as

$$
\tilde{\mathbf{K}}=\left(1+\sqrt{\frac{R}{\mathbf{H P}^{b} \mathbf{H}^{\mathrm{T}}+R}}\right)^{-1} \mathbf{K},
$$

for an individual observation, where $R$ and $\mathbf{H P}^{b} \mathbf{H}^{\mathrm{T}}$ are scalars, while $\mathbf{K}$ and $\tilde{\mathbf{K}}$ are vectors of the same dimension as the model state vector (Whitaker and Hamill 2002).

Distance-dependent covariance localization (Houtekamer and Mitchell 2001; Hamill et al. 2001) is employed to account for the sampling error in the estimation of the background-error covariances. Backgrounderror covariances are forced to taper smoothly to zero $2800 \mathrm{~km}$ away from the observation in the horizontal, and two scale heights $\left[-\ln (\sigma)\right.$, where $\left.\sigma=\left(p / p_{s}\right)\right]$ in the vertical. These values are the same as those used in the MSC ensemble Kalman filter (Houtekamer and Mitchell 2005) operational in 2005. The Blackman window function (Oppenheim and Schafer 1989),

$$
\begin{array}{lll}
A(r)=0.42+0.5 \cos \frac{\pi r}{L}+0.08 \cos \frac{2 \pi r}{L} & r \leq L \\
A(r)=0 & r>L
\end{array}
$$

(where $r$ is the horizontal or vertical distance from the observation and $L$ is the distance at which the covariances are forced to be zero), commonly used in power spectrum estimation, is used to taper the covariances in both the horizontal and vertical. We chose this function instead of the more popular Gaspari-Cohn fifth-order polynomial (Gaspari and Cohn 1999) since it was faster 
to evaluate on our computing platform. The Blackman window function is not formally a spatial correlation function, and therefore the Hadamard product of the Blackman function and a covariance matrix is not guaranteed to be a covariance matrix. This might cause numerical problems in methods that require calculating the inverse of $\mathbf{P}^{b}$, but did not pose any difficulties for the EDA algorithms used here.

The serial processing algorithm employed here is somewhat different than that described in Whitaker et al. (2004). Here, we follow an approach similar to that used in the LETKF (HKS). In the LETKF, each element of the state vector is updated independently, using all of the observations in a local region surrounding that element. All of the observations in the local region are used simultaneously to update the state vector element using the Kalman filter update equations expressed in the subspace of the ensemble. Here, we loop over all of the observations that affect each state vector element, and update that state vector element for each observation using (3) and (4). As suggested by Houtekamer and Mitchell (2005), the forward interpolation operation (the computation of the observation priors, or $\mathbf{H x}^{b}$ ) is precomputed, using the background forecast at several time levels in order to include time interpolation. During the state update (which consists of a loop over all of the elements of the state vector), both the current state vector element and all the observation priors that affect that state vector element are updated. The process can be summarized as follows:

1) Integrate the forecast model forward $t_{a}+0.5 t_{a}$ from the previous analysis time for each ensemble member (where $t_{a}=6 \mathrm{~h}$ is the interval at which observations are assimilated). Save hourly output for each ensemble member from time $t=t_{a}-0.5 t_{a}$ to $t_{a}+$ $0.5 t_{a}$.

2) Compute the observation priors for all observations and ensemble members (i.e., compute $\mathbf{H} \mathbf{x}^{b}$ ). Since the observations occur over the time window from $t_{a}-0.5 t_{a}$ to $t_{a}+0.5 t_{a}$, this involves linear interpolation in time using the hourly model output.

3) Update each element of the model state vector at time $t=t_{a}$. For each element of the state vector (which here includes winds, virtual temperature, surface pressure, and specific humidity), find all of the observations and their associated priors that are "close" to that element (where the definition of close is determined by the covariance localization length scales in the horizontal and vertical). Estimate how much each observation would reduce the ensemble variance for that state element if it were assimilated in isolation, using (4). Sort the observa- tions according to this estimate, so that the observations with the largest expected variance reduction are treated first. Loop over the observations in this order and compute the ratio of the posterior to prior ensemble variance $(F)$ for the current state vector element. If $F \geq 0.99$, skip to the next observation. If $F<0.99$, update the current state vector element for this observation using (3) and (4). Also update all of the close observation priors for this observation (except those for observations that have already been used to update this state element, or those for observations that have been skipped because $F$ exceeded 0.99 , since those observation priors are no longer needed to update the current state vector element). Proceed to the next element of the state vector and repeat. This step can be performed for each state vector element independently in a multiprocessor computing environment, as long as each processor has access to all of the observation priors that can affect the state vector element being updated on that processor. Since the update of the observation priors is done independently on each processor, no communication between processors is necessary.

4) After all elements of the state vector have been updated, adjust the ensemble perturbations to account for unrepresented sources of error (see section 2d). Go to step 1 and repeat for the next analysis time.

Step 3 above includes an adaptive observation thinning algorithm designed to skip observations whose information content is deemed to be negligible. Unlike other recently proposed adaptive thinning algorithms (see Ochotta et al. 2005 and references therein), it uses a flow-dependent estimate of analysis uncertainty to determine if measurements are redundant. If the ratio $F$ (describing the variance of the updated ensemble to the prior ensemble) is close to 1.0 , the observation will have little impact on the ensemble variance for that state vector element. This is likely to be true if a previously assimilated observation has already significantly reduced the ensemble variance for that state element. This approach not only dramatically reduces the computational cost of the state update when observations are very dense, but it also partially mitigates the effect of unaccounted-for correlated observation errors. When observations are much denser than the grid spacing of the forecast model, they are likely resolving scales not represented by the forecast model. Typically, these "errors of representativeness" are accounted for by increasing the value of $R$. However, "representativeness errors" also have horizontal correlations, which are usually not accounted for. Liu and Rabier (2002) 
showed that assimilating dense observations with correlated errors with a suboptimal scheme that ignores those error correlations can actually degrade the analysis (compared with an analysis in which the observations are thinned so that the separation between observations is greater than the distance at which their errors are correlated). The adaptive thinning strategy employed here has the effect of subsampling the observations so that the mean areal separation between observations used to update a given state vector element is increased. The critical value of $F$ used in the thinning (set to 0.99 in this study) can be used to control this separation, with smaller values of $F$ resulting in larger separation distances. Adaptive thinning may actually improve the analysis in situations where there are significant unaccounted-for error correlations between nearby observations. If correlations between nearby observations are properly accounted for in $\mathbf{R}$, there should be no benefit to this type of adaptive thinning, other than to reduce the computational cost of computing the analysis increment. There are some potential problems with this adaptive thinning algorithm. For one, since the observation selection proceeds serially, starting with observations that will have the largest predicted impact on ensemble variance, it is possible that a less accurate observation (e.g., a satellite-derived wind) will be selected over a more accurate one (say, a radiosonde wind), if the less accurate one is predicted to have a larger impact on the ensemble variance. Although in principle the less accurate observation should be of greater utility in this situation, under some circumstances one may prefer to assimilate the "best," or most accurate observation. Second, since each state vector element is updated independently, it is possible that quite different sets of observations will be selected to update adjacent grid points, potentially resulting in discontinuities in the analyzed fields.

The main advantage of this approach over the serial processing algorithm used in Whitaker et al. (2004) is that it is more easily parallelized on massively parallel computers. Step 3, the update for each element of the state vector, can be performed independently for each element of the state vector. Therefore, the state vector can be partitioned arbitrarily, and each partition can be updated on a separate processor. No communication between processors is necessary during the state update. However, there is a significant amount of redundant computation associated with the update of the observation priors in step 3. This is because nearby state vector elements are influenced by overlapping sets of observations, so that different processors must update nearly identical sets of observation priors.

Since the observation network is very inhomoge- neous, some elements of the state vector can be influenced by a much larger number of observations than other elements. For example, state vector elements in the mesosphere or near the South Pole will be influenced by very few observations, while those in the lower troposphere over Europe or North America will be influenced by a much larger number of observations. To alleviate load imbalances that may occur if some processors are updating state vector elements that are "observation rich," while others are updating state vector elements that are "observation poor," the latitude and longitude indices of the model state vector are randomly shuffled before being assigned to individual processors. This means that all of the observations and observation priors must be distributed to all processors.

The algorithm used here has a couple of potential advantages over the LETKF. The serial processing algorithm allows for adaptive thinning of observations, in a way that is not easily achievable in the LETKF framework where all of the observations are assimilated simultaneously. This may prove to be a benefit when observation errors are significantly correlated, but assumed to be uncorrelated. However, if observation error correlations are accounted for in $\mathbf{R}$, the LETKF may be preferable, since serial processing becomes significantly more complicated when $\mathbf{R}$ is not diagonal [the ensemble must be transformed into a space in which $\mathbf{R}$ is diagonal; Kaminski et al. (1971)]. Since the LETKF is performed in the subspace of the ensemble, covariance localization, as it is traditionally applied, is problematic. HKS have suggested that the effect of covariance localization may be mimicked in the LETKF by increasing the value of $\mathbf{R}$ as a function of the distance from the state vector element being updated. This "observationerror localization," tested in the LETKF by Miyoshi and Yamane (2007), allows the influence of observations to decay smoothly to zero at a specified distance from an analysis grid point without increasing the effective number of degrees of freedom in the ensemble (as is the case when covariance localization is applied to $\left.\mathbf{P}^{b}\right)$. Our code allows the LETKF algorithm or the serial processing algorithm to be activated by a run-time switch. In section $3 d$, we will compare assimilation results using the LETKF with observation-error localization (using all available observations) to those obtained using the serial algorithm with "traditional" covariance localization (and adaptive observation thinning).

\section{d. Accounting for system errors}

As discussed in Houtekamer and Mitchell (2005), ensemble data assimilation systems are suboptimal because of (i) sampling error in the estimation of background-error covariances, (ii) errors in the specification 
of the observation error statistics, (iii) errors in the forward interpolation operator, (iv) the possible nonGaussianity of forecast and observation errors, and (v) errors in the forecast model that have not been accounted for. The effects of all of these are mixed together as the assimilation system is cycled, so a quantitative assessment of their relative impacts is difficult. Their net effect on an ensemble data assimilation system is to introduce a bias in the error covariances, such that they are too small and span a different eigenspace than the forecast errors. As a result, various ad hoc measures must be taken to avoid filter divergence, including the following:

- Distance-dependent covariance localization (Houtekamer and Mitchell 2001; Hamill et al. 2001) is usually employed to combat (i), and is used here.

- Adaptive thinning of observations as previously described, or the construction of "superobservations" (Daley 1991) are examples of methods used to combat (ii).

- The effects of (iii) are often accounted for indirectly by increasing the value of the observation error to account for the error of representativeness, while keeping $\mathbf{R}$ diagonal. However, this approach does not fully account for the spatially correlated part of the error in the forward operator.

- The Kalman filter is a special case of Bayesian state estimation that assumes normal error distributions, so the effects of (iv) can only be dealt with by relaxing that assumption, which implies a redefinition of the update equations [(1)-(5)].

Item v, model error, is the most difficult to deal with. In fact, as pointed out by Houtekamer and Mitchell (2005), this category is often used as a catchall for the effects of all misrepresented error sources that are accumulated as the data assimilation system is cycled and may more properly be termed system error. We have tested three relatively simple methods for accounting for system error in our data assimilation system. These are (i) covariance, or multiplicative inflation (Anderson and Anderson 1999); (ii) additive inflation (Houtekamer and Mitchell 2005); and (iii) relaxation-to-prior (Zhang et al. 2004). These methods are meant to account for biases in the second moment of the ensemble, and not the mean. Systematic model errors can cause the ensemble mean to be biased. Schemes to correct for systematic model errors in EDA have been proposed (Baek et al. 2006; Keppenne et al. 2005), but in the experiments shown here we have not accounted for biases in the ensemble mean first guess. Each of the three methods used here is applied to the posterior ensemble, after the computation of the analysis increment and before running the forecasts to be used as the first guess for the next state update. This is done to more easily accommodate time interpolation in the forward operator. Since time interpolation requires the use of the first-guess ensemble at several forecast times, if the system error parameterization were applied to the prior ensemble instead of the posterior ensemble, the parameterization would have to be applied at each forecast time used in the time interpolation. Applying these parameterizations to the posterior ensemble is justified by our reinterpretation of the model error as a system error reflecting an accumulation of errors arising from several components of the data assimilation system, not just the forecast model (Houtekamer and Mitchell 2005). Another method for dealing with model error, which we have not investigated here, involves explicitly accounting for the uncertainty in the forecast model by using a multimodel ensemble (Meng and Zhang 2007; Houtekamer et al. 1996).

The first method for treating system error, known as covariance, or multiplicative inflation (Anderson and Anderson 1999), simply inflates the deviations from the ensemble mean by a factor $r>1.0$ for each member of the ensemble. We have found that different inflation factors were required in the Northern and Southern Hemispheres and in the troposphere and stratosphere because of the large differences in the density of the observing networks. In the limit that there are no observations influencing the analysis in a given region, it is easy to envision how inflating the ensemble every analysis time can lead to unrealistically large ensemble variances, perhaps even exceeding the climatological variance (Hamill and Whitaker 2005). Here, we use an inflation factor of $r=1.30$ in the Northern Hemisphere (poleward of $25^{\circ} \mathrm{N}$ ) at $\sigma=1, r=1.18$ in the Southern Hemisphere (poleward of $25^{\circ} \mathrm{S}$ ) at $\sigma=1$, and $r=1.24$ in the tropics (between $15^{\circ} \mathrm{S}$ and $15^{\circ} \mathrm{N}$ ) at $\sigma=1$. The values vary linearly in latitude in the transition zone between the tropics and extratropics. In the vertical, the values of $r$ taper smoothly from their maximum values at the surface to 1.0 at six scale heights $[-\ln (\sigma)=6]$. The Blackman function, (6), is used to taper the inflation factor in the vertical.

The second method for treating system error is additive inflation. In the standard Kalman filter formulation, model error is parameterized by adding random noise with a specified covariance structure in space, and zero correlation in time, to the background-error covariances after those covariances are propagated from the previous analysis time using a linear model. Applying this approach to an ensemble filter involves adding random perturbations, sampled from a distribution with known covariance statistics, to each ensemble member. 
We call this technique, which is currently used by the MSC in their operational ensemble data assimilation system, additive inflation. MSC uses random samples from a simplified version of the static covariance model used in their variational analysis. Here, we have chosen to use scaled random differences between adjacent 6-hourly analyses from the NCEP-National Center for Atmospheric Research (NCAR) reanalysis (Kistler et al. 2001). The reason for this choice is that 6-h tendencies will emphasize baroclinically growing, synopticscale structures in middle latitudes, while 3DVAR covariance structures tend to be larger scale and barotropic. Analysis systems that only use information about observations prior to the analysis time tend to concentrate the error in the subspace of growing disturbances (e.g., Pires et al. 1996), so our use of 6-h tendencies is based on the assumption that the accumulated effect of unrepresented errors in the data assimilation system will be concentrated in dynamically active, growing structures. This choice of additive inflation was shown to work well in a study of the effect of the model error on an ensemble data assimilation in an idealized general circulation model (Hamill and Whitaker 2005). The random samples are selected from the subset of 6-hourly reanalyses for the period 1971-2000 that are within 15 days of the calendar date of the analysis time. The differences between these randomly selected, adjacent analysis times are scaled by 0.33 before being added to each member of the posterior, or analysis ensemble.

The third method for treating system error, the relaxation-to-prior method, was proposed by Zhang et al. (2004) as an alternative to covariance inflation. The name refers to the fact that it relaxes the analysis perturbations back toward the prior perturbations independently at each analysis point via

$$
\mathbf{x}^{\prime a} \leftarrow(1-\alpha) \mathbf{x}^{\prime a}+\alpha \mathbf{x}^{\prime b} .
$$

The adjustment is performed after the state update, and the modified analysis perturbations are then integrated forward to form the background ensemble for the next analysis time. The advantage of this approach relative to covariance inflation is that the ensemble perturbations are only modified where observations have an effect on the analysis, thereby avoiding the tendency for ensemble variance to increase without bound where observations have no influence. Zhang et al. (2004) used a value of 0.5 for their perfect model convectivescale ensemble data assimilation experiment, but Meng and Zhang (2007) found that increasing that value to 0.7 or higher was beneficial when significant model error was present. Here, we found that a value of 0.88 produced the best results. A value of 0.88 means that the weight given to the prior ensemble perturbations is $88 \%$, and only $12 \%$ weight is given to the analysis perturbations computed from the state update (4).

\section{Results}

Three experiments were performed with the EDA. All of the experiments used the parameter settings given in the previous section for covariance localization and adaptive observation thinning. Only the parameterization of the system error was changed. The EDAmultinf experiment used multiplicative covariance inflation, the EDA-addinf used additive inflation (derived from random samples of 6-h differences from the NCEP-NCAR reanalysis), and the EDA-relaxprior experiment used the relaxation-to-prior method to increase the variance in the posterior ensemble. The parameter settings used for these three experiments are as given in the previous section. Assimilations were performed for the period 0000 UTC 1 January to 0000 UTC 10 February 2004. Forecasts initialized from the ensemble mean analyses for each of these experiments are compared with forecasts initialized from the NCEPBenchmark analyses. The forecasts were run at T62L28 resolution (the same resolution used in the data assimilation cycle), and are verified against observations and the NCEP-Operational analyses. The initial ensemble for the EDA assimilation runs consisted of a random sample of 100 operational GDAS analyses from February 2004. The NCEP-Benchmark assimilation run was started from the operational GDAS analysis at 0000 UTC 1 January 2004. After an initial spinup period of 1 week, verification statistics were computed for 125 forecasts initialized every $6 \mathrm{~h}$ from 0000 UTC 8 January to 0000 UTC 8 February 2004.

\section{a. Verification using observations}

Three different subsets of observations were used for forecast verifications: marine surface pressure observations, upper-tropospheric (300-150 hPa) aircraft report (AIREP) and pilot report (PIREP) wind observations, and radiosonde profiles of wind and temperature. Note that this subset is only a small fraction of the total number of observations assimilated. Figure 1 shows the spatial distribution of these observation types for a typical day. Radiosonde observations mainly sample the continental regions of the Northern Hemisphere. Marine surface pressure observations sample the ocean regions of both hemispheres, but are densest in the North Atlantic. Aircraft observations are mainly confined to the western half of the Northern Hemisphere, but sample 
(A) Radiosonde Locations

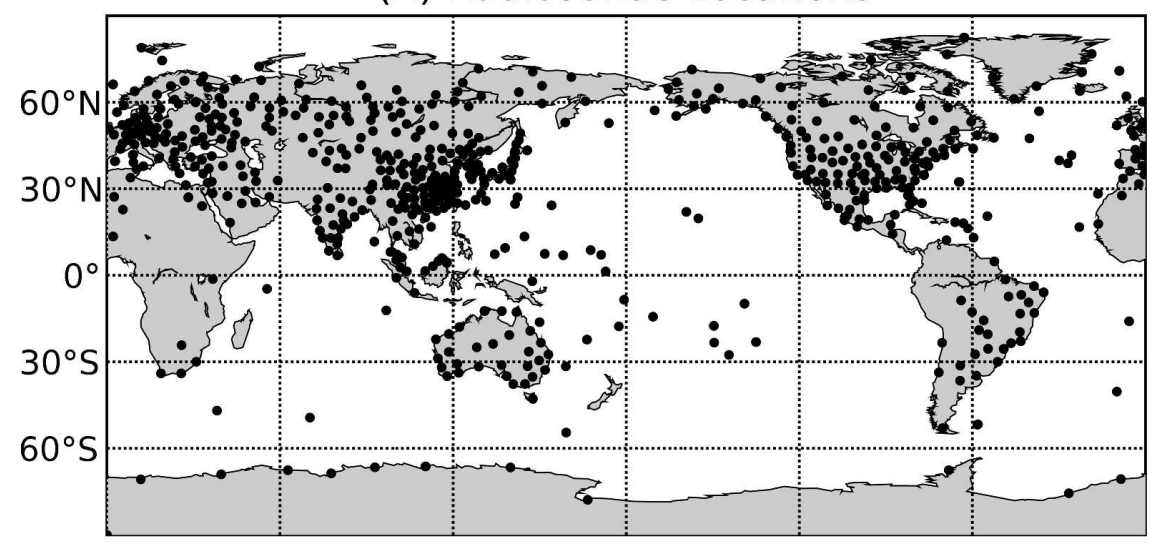

(B) Aircraft Locations

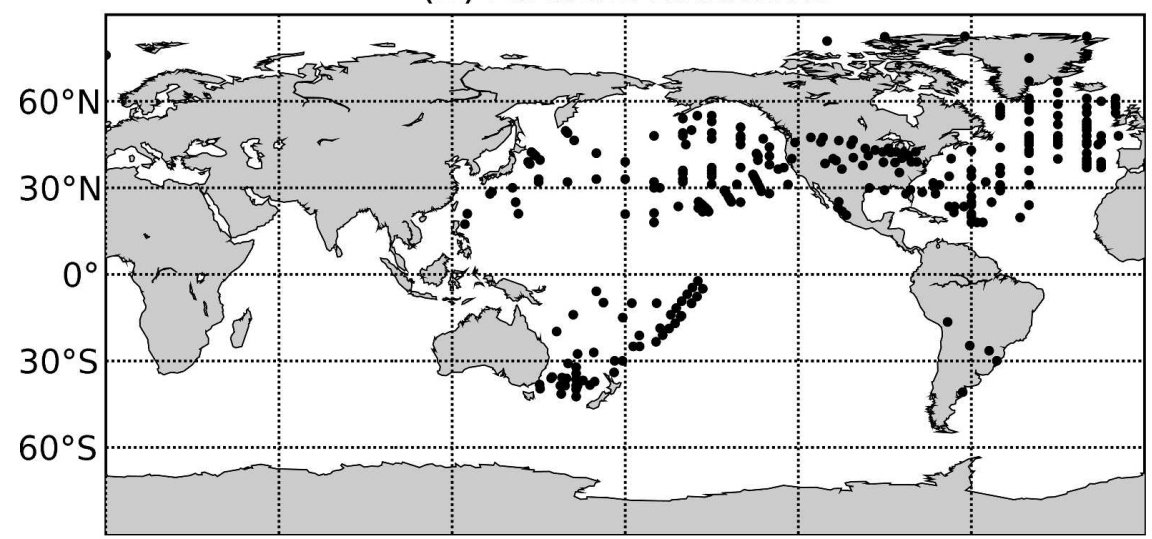

(C) Marine Surface Pressure Locations

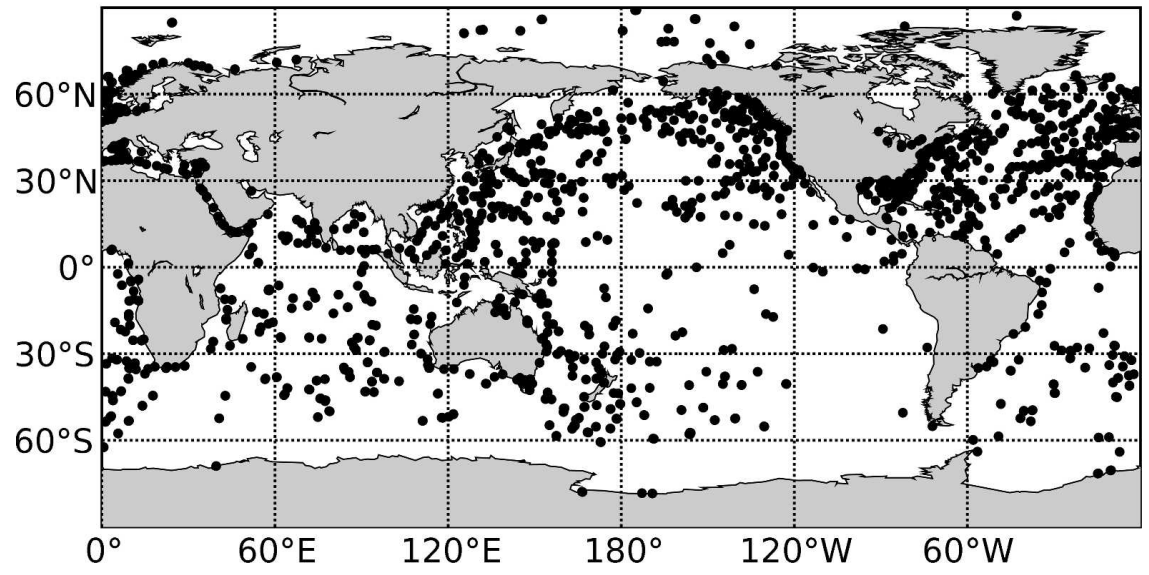

FIG. 1. Locations of (a) radiosonde observations, (b) aircraft observations (AIREP and PIREP observations between 300 and $150 \mathrm{hPa}$ ), and (c) surface marine (ship and buoy) pressure observations for 0000 and 1200 UTC 10 Jan 2004.

both the continents of North America and Europe and the Pacific and Atlantic Ocean basins.

Table 1 shows the root-mean-square (RMS) fit of 48-h forecasts to marine surface pressure and upper- tropospheric aircraft meridional wind observations. With the possible exception of the EDA-relaxprior forecasts of meridional wind, the EDA-based forecasts fit the observations significantly better than the NCEP- 
TABLE 1. Fits of 48-h forecasts initialized from 0000, 0600, 1200, and 1800 UTC EDA ensemble mean and NCEP-Benchmark analyses to aircraft (AIREP and PIREP) observations between 300 and $150 \mathrm{hPa}$ and surface marine (ship and buoy) pressure observations from 8 Jan to 8 Feb 2004. Three different EDA analyses are shown, each employing a different method for parameterizing system error (see text for details). The confidence level (\%) for the difference between each of the EDA results and the NCEP-Benchmark result is also given. This confidence level is computed using a two-tailed $t$ test for the difference between means of paired samples, taking into account serial correlations in the data.

\begin{tabular}{lllcr}
\hline \hline & EDA-addinf & EDA-multinf & EDA-relaxprior & NCEP-Benchmark \\
\hline Aircraft meridional wind $\left(\mathrm{m} \mathrm{s}^{-1}\right)$ & $7.57(99 \%)$ & $7.54(>99 \%)$ & $7.68(91 \%)$ & 7.78 \\
Surface marine pressure $(\mathrm{hPa})$ & $3.13(>99 \%)$ & $3.24(>99 \%)$ & $3.21(>99 \%)$ & 3.40 \\
\hline
\end{tabular}

Benchmark forecasts. The significance level from a paired sample $t$ test (Wilks 2006, p. 455) for the difference between the mean EDA forecast fits and the mean NCEP-Benchmark forecast fits is also given in Table 1 for each of the EDA experiments. This significance test takes into account serial correlations in the data by modeling the sample differences as a first-order autoregressive process. The significance level is then computed using an "effective sample size" $n^{\prime}=n(1-r)$ / $(1+r)$ (Wilks 2006, p. 144), where $n=125$ is the total sample size and $r$ is the lag-1 autocorrelation of the forecast error differences. The EDA-addinf forecasts of surface pressure appear to fit the observations better than the EDA-multinf and EDA-relaxprior forecasts, although the differences between the EDA experiments are not significant at the $99 \%$ confidence level.

Figure 2 shows the global RMS fit of 6- and 48-h forecasts to radiosonde profiles of meridional wind and temperature for each of the experiments. With the exception of the EDA-relaxprior forecasts, the EDAbased forecasts fit the radiosonde observations at most levels better than the NCEP-Benchmark forecasts. Aggregating all of the observations between $\sigma=0.9$ and $\sigma=0.07$, the difference between EDA-addinf and EDA-multinf and the NCEP-Benchmark forecasts is significant at the $99 \%$ level. The EDA-relaxprior forecasts are not significantly closer to the radiosonde observations than are the NCEP-Benchmark forecasts. The 48-h EDA-addinf forecasts generally have the lowest error, although the difference between the EDAaddinf and EDA-multinf forecasts is not statistically significant at the $99 \%$ level.

Table 2 shows the RMS fit of 48 -h forecasts to marine surface pressure observations for the Northern Hemisphere and the Southern Hemisphere separately. We have only stratified the results by hemisphere for marine surface pressure because the other observation types are primarily concentrated in the Northern Hemisphere (Fig. 1). The difference between the fit of EDA forecasts and NCEP-Benchmark forecasts to marine surface pressure observations is larger in the Southern Hemisphere extratropics than in the Northern Hemi- sphere extratropics (Table 2). This result agrees with previous studies using EDA systems in a perfect-model context (Hamill and Snyder 2000) and using real observations characteristic of observing networks of the early twentieth century (Whitaker et al. 2004), which have shown that the flow-dependent background-error covariances these systems provide have the largest impact when the observing network is sparse.

\section{b. Verifications using analyses}

When comparing forecasts and analyses from different centers, the standard practice in the operational weather prediction community has been to verify each forecast against its own analysis, that is, the analysis generated by the same center. The problem with this approach is that an analysis can perform well in this metric if the assimilation completely ignores the observations. Here, we have the luxury of having an independent, higher quality analysis to verify against, the NCEP-Operational analysis. Since this analysis was run at four times higher resolution and used a large set of observations (including the satellite radiances), we expect it to be significantly better. We have verified that this is indeed the case ${ }^{3}$ especially in the Southern Hemisphere where satellite radiances have been found to have the largest impact on analysis quality (e.g., Derber and Wu 1998; Simmons and Hollingsworth 2002).

Figure 3 shows vertical profiles of 48 -h geopotential height and meridional wind forecast errors for both the Northern Hemisphere and Southern Hemisphere for forecasts initialized from analyses produced by each of the EDA experiments and the NCEP-Benchmark experiment. Results for the zonal wind are very similar to the meridional wind (not shown). For the most part, forecasts from each of the EDA experiments track the NCEP-Operational analysis at $48 \mathrm{~h}$ better than the

\footnotetext{
${ }^{3}$ Results are obtained by running forecasts from the operational analysis with the same T62L28 version of the forecast model and comparing those forecasts with radiosonde and marine surface pressure observations.
} 

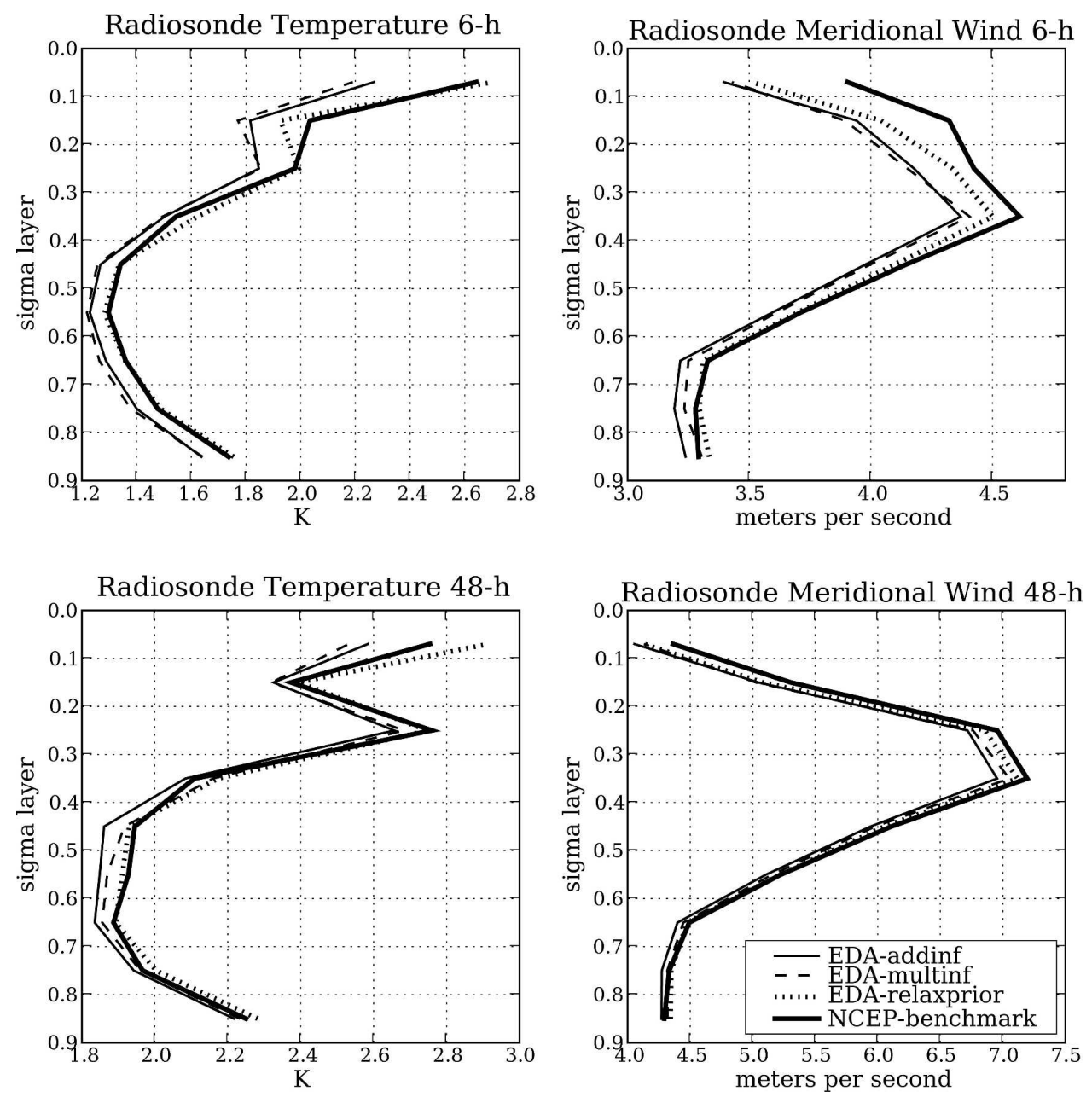

FIG. 2. RMS fits of 6- and 48-h forecasts initialized from 0000, 0600, 1200, and 1800 UTC EDA ensemble mean and NCEP-Benchmark analyses to radiosonde observations from 8 Jan to 8 Feb 2004. Three different EDA analyses are shown, each employing a different method for parameterizing system error (see text for details). Note that the ordinate is a sigma layer, not a pressure level, since the calculations were done in sigma coordinates. There is one data point for each interval of width $\Delta \sigma=0.1$, beginning at $\sigma=0.9$, representing all of the observations in each layer.

NCEP-Benchmark forecasts. The lone exception is the EDA-relaxprior forecasts of meridional wind in the Northern Hemisphere. All of the other EDA forecasts are more skillful than the NCEP-Benchmark forecasts, and these differences are significant at the $99 \%$ level at $500 \mathrm{hPa}$, using the paired-sample $t$ test for serially correlated data described previously. The EDA-addinf performs better overall than the EDA-multinf and EDA-relaxprior experiments, although the differences are only statistically significant at the $99 \%$ level in the Northern Hemisphere.

The improvement seen in the EDA experiments relative to the NCEP-Benchmark is especially dramatic in the Southern Hemisphere, where Fig. 4 shows that it is

TABLE 2. As in Table 1, but for surface marine pressure observations in the Northern Hemisphere and Southern Hemisphere extratropics (poleward of $20^{\circ} \mathrm{N}$ and $20^{\circ} \mathrm{S}$ ).

\begin{tabular}{llllr}
\hline \hline & EDA-addinf & EDA-multinf & EDA-relaxprior & NCEP-Benchmark \\
\hline NH extratropics $(\mathrm{hPa})$ & $3.29(>99 \%)$ & $3.43(75 \%)$ & $3.39(85 \%)$ & 3.48 \\
SH extratropics $(\mathrm{hPa})$ & $3.21(>99 \%)$ & $3.24(>99 \%)$ & $3.23(>99 \%)$ & 3.75 \\
\hline
\end{tabular}


N. Hem. Geopotential Height

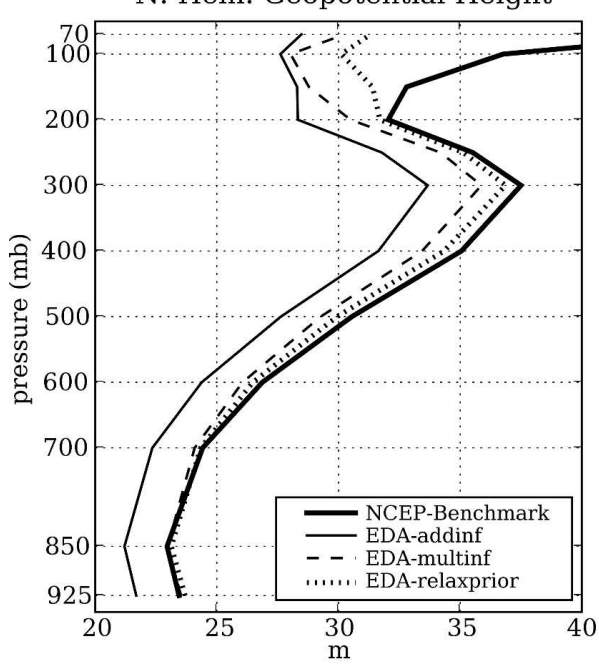

N. Hem. Meridional Wind

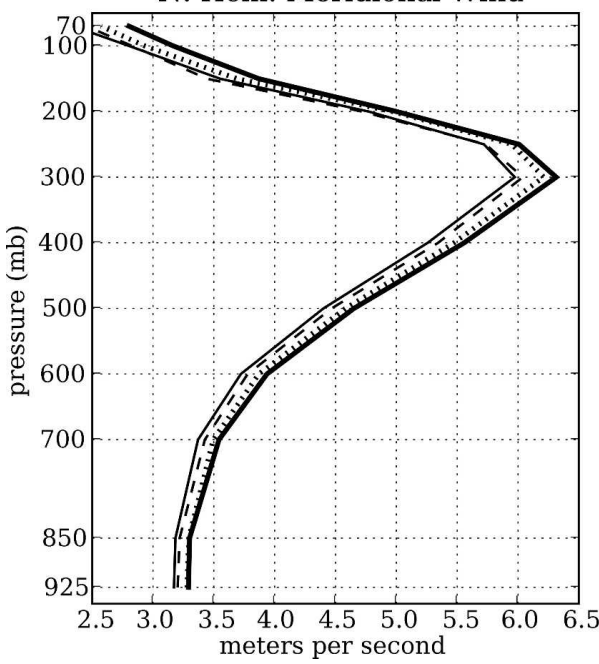

S. Hem. Geopotential Height

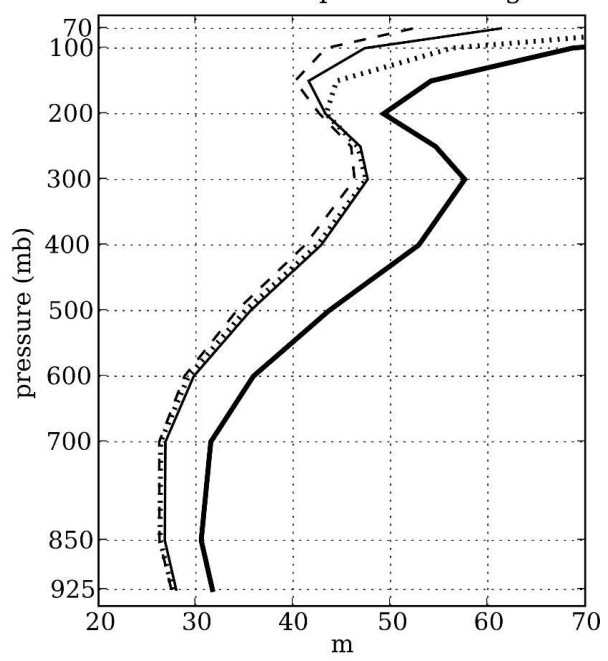

S. Hem. Meridional Wind

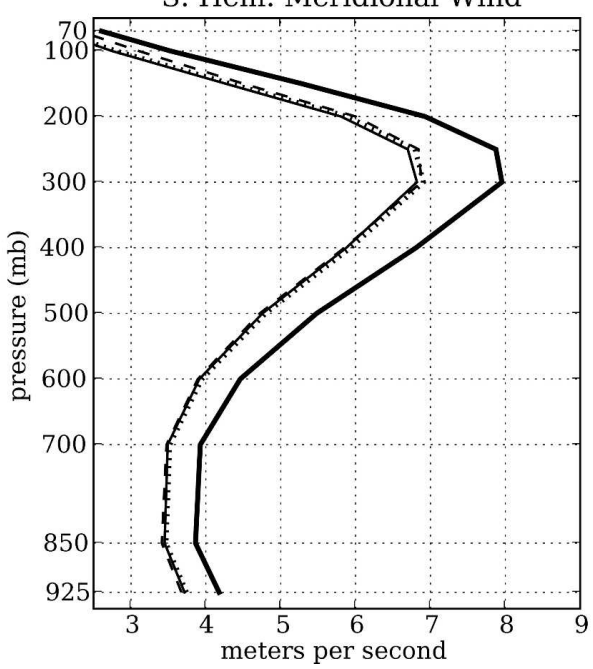

FIG. 3. Vertical profiles of 48-h forecast error (measured relative to the NCEP-Operational analysis). Values for the Northern Hemisphere (poleward of $20^{\circ} \mathrm{N}$ ) and the Southern Hemisphere (poleward of $20^{\circ} \mathrm{S}$ ) are shown for geopotential height and meridional wind, for each of the EDA experiments and the NCEP-Benchmark experiment.

equivalent to $24 \mathrm{~h}$ of lead time (in other words, 48-h forecasts initialized from the EDA-addinf analyses are about as accurate as 24-h forecasts initialized from the NCEP-Benchmark 3DVAR analysis). In the Northern Hemisphere, the advantage that the EDA-addinf based forecasts have over the NCEP-Benchmark forecasts is closer to $6 \mathrm{~h}$ in lead time. This is further evidence that flow-dependent covariances are most important in data-sparse regions. This is illustrated for a specific case in Fig. 5, which shows the 500-hPa analyses produced by the NCEP-Benchmark, NCEP-Operational, and EDA-addinf analysis systems for 0000 UTC 3 February 2004. The difference between the NCEP-Benchmark and EDA-addinf analyses is especially large (greater than $100 \mathrm{~m}$ ) in the trough off the coast of Antarctica near $120^{\circ} \mathrm{W}$, where the EDA-addinf is much closer to the NCEP-Operational analysis. This region corresponds to the most data-sparse region of the Southern Hemisphere, as can be seen from the distribution of marine surface pressure observations in Fig. 1. The EDA system is clearly able to extract more information from the sparse Southern Hemisphere observational network than the NCEP-Benchmark system, and compares favorably to the higher-resolution operational analysis, which utilized more than an order of magnitude more observations in this region (by assimilating satellite radiance measurements).

Since in situ humidity measurements are quite sparse 

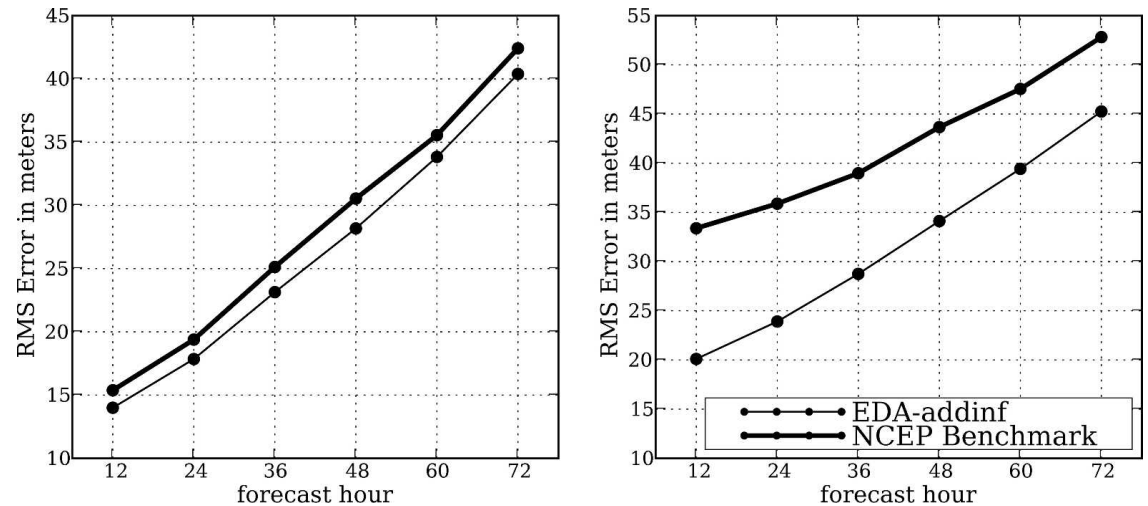

FIG. 4. The 500-hPa geopotential height forecast error (measured relative to the NCEPOperational analysis) as a function of forecast lead time, for the (left) Northern and (right) Southern Hemisphere extratropics (the region poleward of $20^{\circ}$ ). The thinner curve is for forecasts initialized from the ensemble mean EDA-addinf analysis, and the thicker curve is for forecasts initialized from the NCEP-Benchmark analysis.

(A) EDA-addinf Analysis

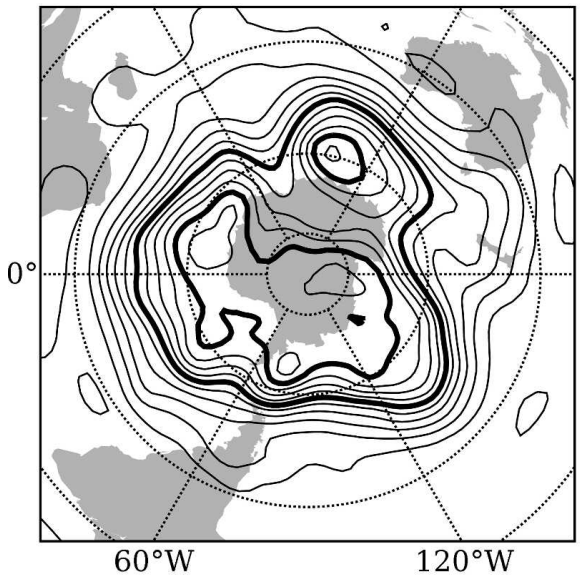

(C) NCEP-Operational Analysis

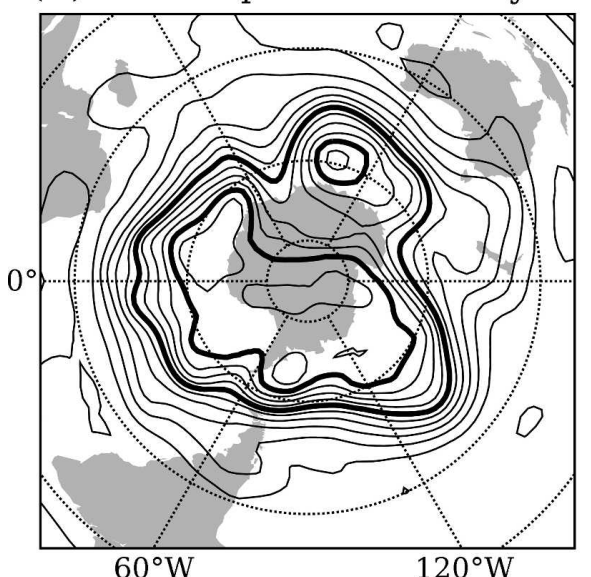

(B) NCEP-Benchmark Analysis

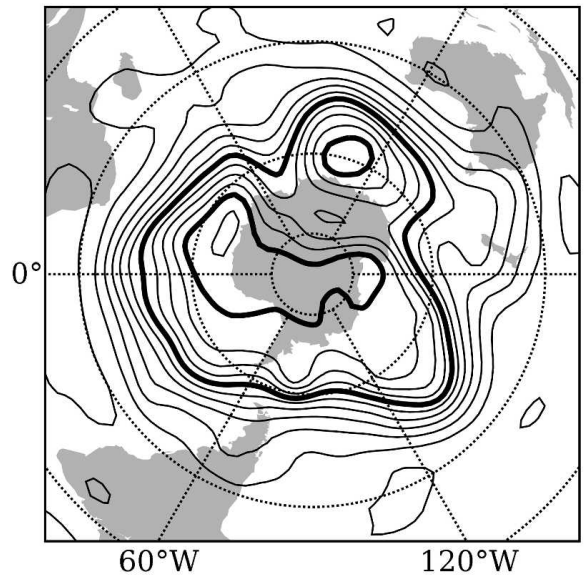

(D) EDA-addinf minus NCEP-Benchmark

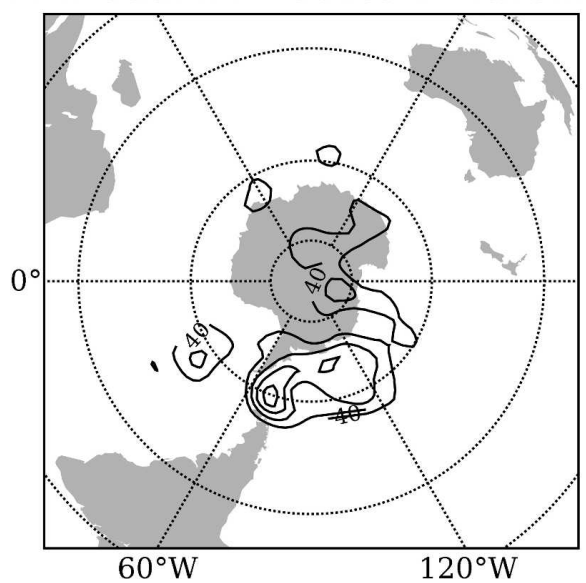

FIG. 5. The 0000 UTC 3 Feb 2004 Southern Hemisphere 500-hPa geopotential height analyses for the (a) EDA-addinf, (b) NCEP-Benchmark, and (c) NCEP-Operational analysis systems. (d) The difference between the EDA-addinf and NCEP-Benchmark analyses. The contour interval is $75 \mathrm{~m}$ in (a)-(c) and $40 \mathrm{~m}$ in (d). The 5125and 5425-m contours are emphasized in (a)-(c). All contours in (d) are negative. 

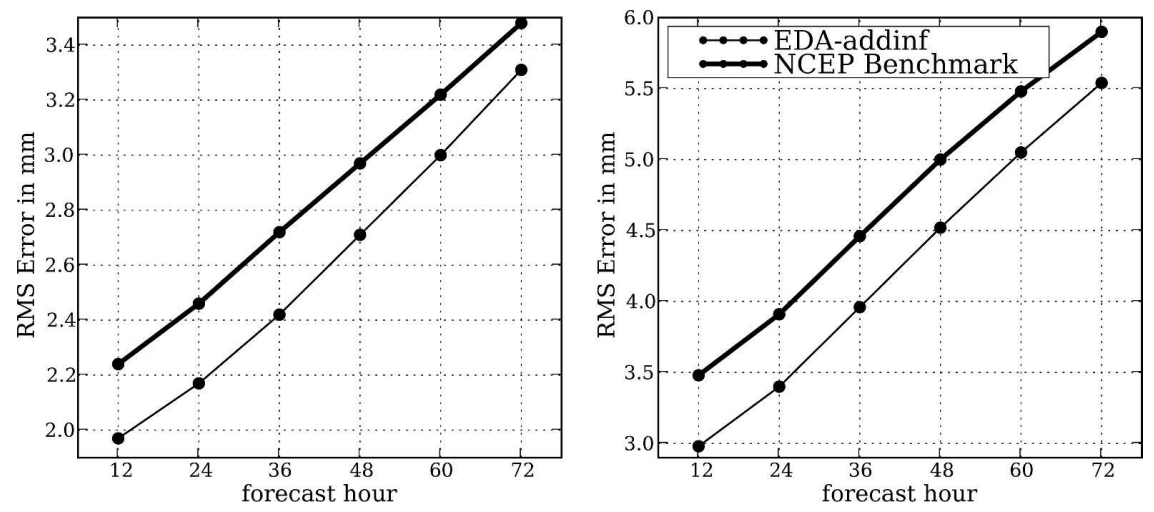

FIG. 6. As in Fig. 4, but for total precipitable water (mm).

(they are only available from radiosondes in the experiments presented here), one might expect the ensemblebased data assimilation to show a clear advantage over 3DVAR for the moisture field. Figure 6 shows the RMS error of total precipitable water forecasts for EDA-addinf and NCEP-Benchmark based forecasts. These forecasts are verified against the higherresolution NCEP operational analysis, which includes remotely sensed humidity measurements. The EDAbased forecasts have approximately a 12-h advantage in lead time relative to the 3DVAR-based forecasts, in both the Northern and Southern Hemisphere extratropics. The EDA systems can utilize nonmoisture obser- vations to make increments to the first-guess moisture field through cross covariances between the moisture field and the other state variables provided by the ensemble. In the NCEP 3DVAR system, only observations of humidity can increment the first-guess moisture field, since the static covariance model does not include cross covariances between humidity and other state variables. Figure 7 shows the increment of total precipitable water implied by a single observation of surface pressure (show by the black dot) in the EDA-addinf system for 0000 UTC 30 January 2004. The EDA system is able to take into account the dynamical relationship between the strength of the low center and the

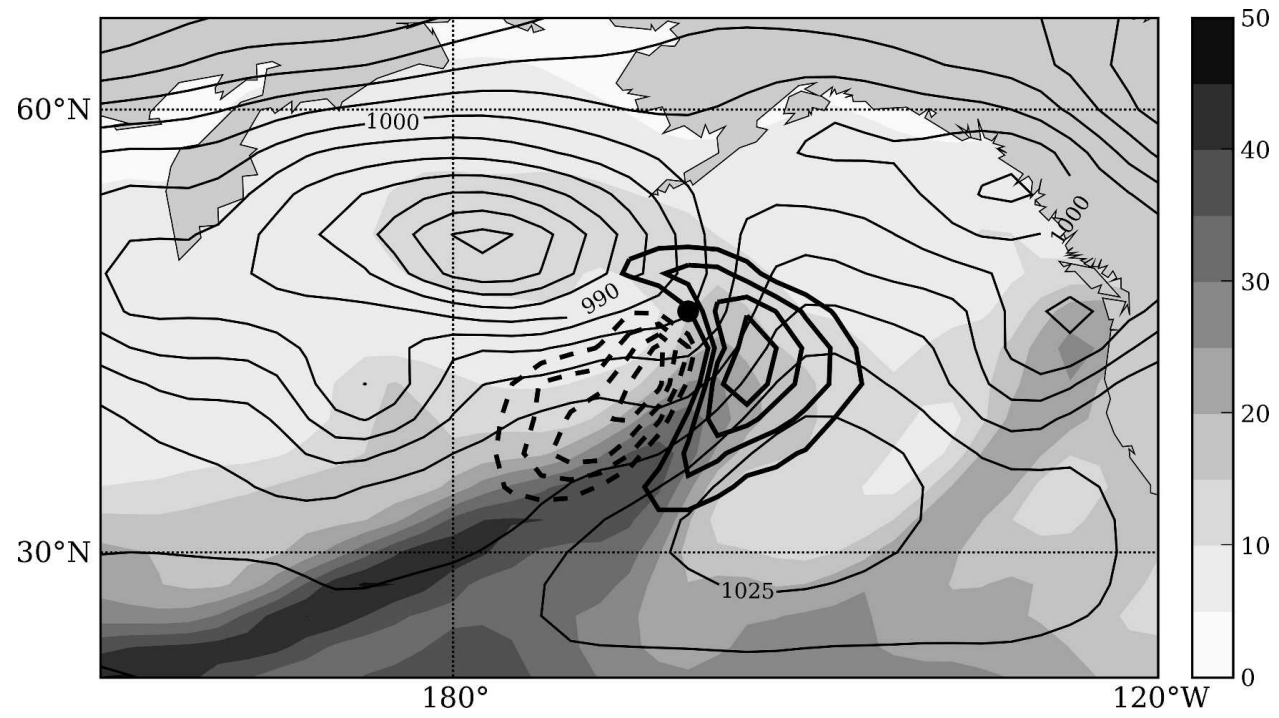

FIG. 7. Precipitable water analysis increment (thick black contour lines, contour interval of $0.5 \mathrm{~mm}$, dashed lines negative, zero contour suppressed) from the EDA-addinf system at 0000 UTC 30 Jan 2004 associated with a surface pressure observation located at the black dot at the center of the map. The surface pressure observation is $1 \mathrm{hPa}$ lower than the ensemble mean first-guess forecast (thin black contours, interval of $5 \mathrm{hPa}$ ). The gray-shaded field is the ensemble mean first-guess precipitable water (scale on right denotes contour levels). 
amplitude of the moisture plume ahead of the cold front. An accurate, flow-dependent treatment of these dynamical relationships, as represented by the crossvariable covariances in the ensemble, is particularly important in the analysis of unobserved variables. Another example of this was given in the context of the assimilation of radar reflectivity into a cloud-resolving model by Snyder and Zhang (2003). In that study, the cross covariances between the predicted radar reflectivity and the other model state variables (winds, temperature, moisture, and condensate) provided by the ensemble were crucial in obtaining an accurate analysis when only radar reflectivity was observed.

\section{c. Ensemble consistency}

If the EDA system is performing optimally, the innovation covariances should satisfy

$$
\left\langle\left(\mathbf{y}^{o}-\mathbf{H} \overline{\mathbf{x}}^{b}\right)\left(\mathbf{y}^{o}-\mathbf{H} \overline{\mathbf{x}}^{b}\right)\right\rangle=\mathbf{H P}^{b} \mathbf{H}^{\mathrm{T}}+\mathbf{R}
$$

(Houtekamer et al. 2005), where the angle brackets denote the expectation value. Here, we compare the diagonal elements of the matrices on the left- and righthand sides of (8), computed for radiosonde observations in the EDA experiments. If the magnitudes of those diagonal elements are similar, the ensemble is said to be consistent; that is, the innovation variances are consistent with the background and observation error variances at the observation locations. This primarily reflects the degree to which the parameterization of system error has been tuned, although with the small number of parameters used here to represent system error, it will be difficult if not impossible to achieve a perfect match, unless the parameterization of the system error itself is very accurate.

Figure 8 shows the square root of the ensemble spread plus the observation-error variance [the diagonal of the right-hand side of (8)] at radiosonde locations for the three EDA experiments. This quantity can be regarded as the "predicted" innovation standard deviation, since if (8) is satisfied, the two quantities will be the same. For the purposes of this discussion, we will assume that any disagreement between the left- and right-hand sides of (8) is due to deficiencies in the background-error covariance, and not the observation-error covariance. The actual innovation standard deviation (or the root-mean-square fit) is shown in Fig. 8 only for the EDA-addinf experiment because the radiosonde fits for the other EDA experiments are quite similar. For both temperature and meridional wind, the ensemble spread in the lower troposphere is deficient for all three EDA experiments. This means that none of the EDA systems are making optimal use of the radio-
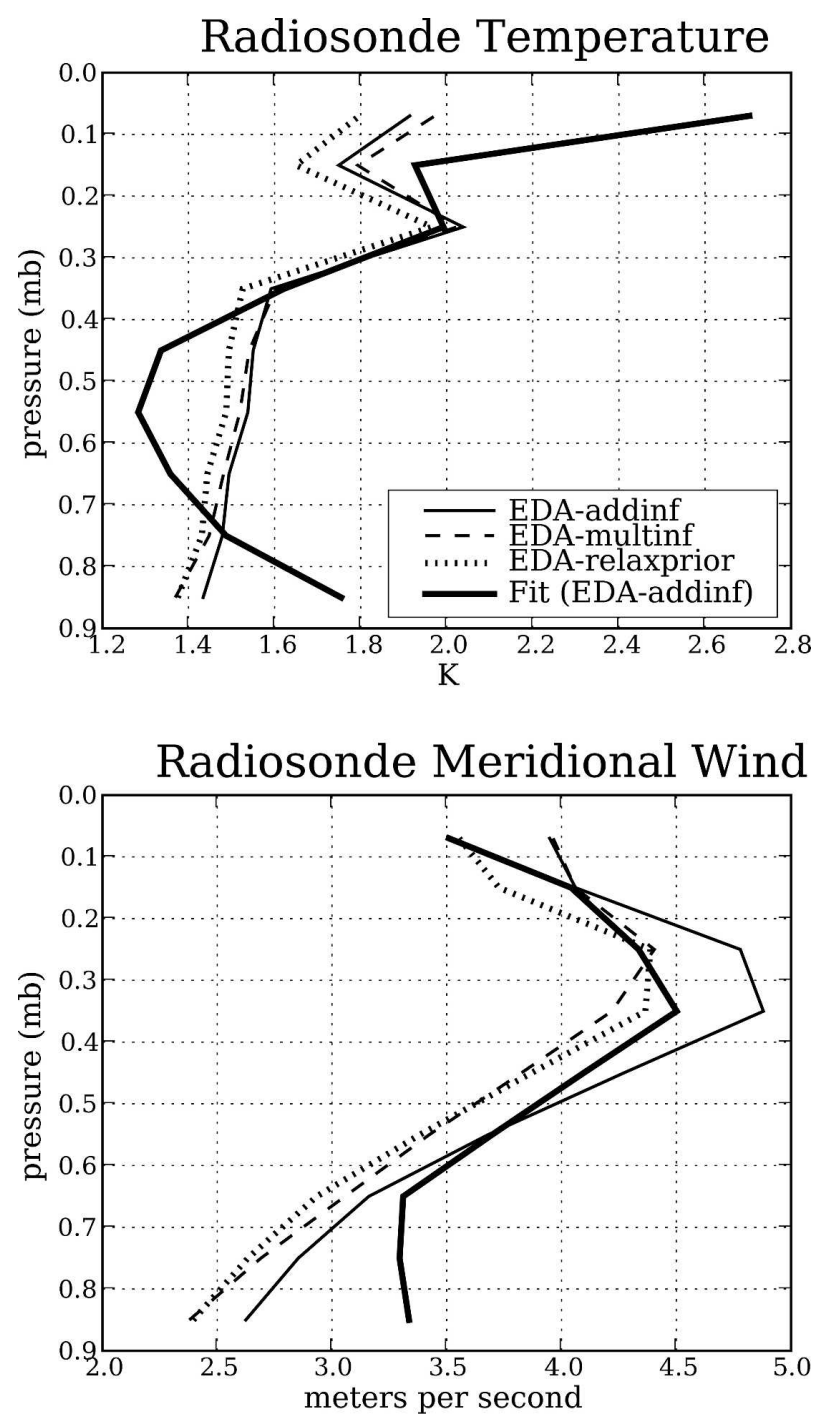

FIG. 8. Square root of ensemble spread plus observation error variance at radiosonde locations for 6-h EDA forecasts initialized at 0600 and 1800 UTC from 8 Jan to 8 Feb 2004. Three different EDA ensembles are shown, each employing a different method for parameterizing system error (see text for details). The RMS fit of the 6-h EDA-addinf ensemble mean forecast to the radiosonde observations is also shown (heavy solid curve).

sondes in the lower troposphere. In particular, they are weighting the first guess too much.

Houtekamer et al. (2005) showed diagnostics similar to these for the MSC implementation of the ensemble Kalman filter (their Fig. 5). The actual innovation standard deviations for their implementation are quite similar to ours, but the predicted innovation standard deviations appear to match the actual values more closely, particularly in the lower troposphere. In the MSC implementation, the system error is additive and is derived from random samples drawn from a simplified 
version of their operational background-error covariance model. The MSC operational covariance itself has been tuned so that innovation statistics for the radiosonde network are consistent with the observation and background error variance. Therefore, it is perhaps not surprising that the vertical structure of the predicted innovation standard deviation more closely matches the actual radiosonde innovations. In all three of our system error parameterizations, there is only one parameter that can be tuned (in the case of multiplicative inflation, this parameter can be tuned separately in the Northern Hemisphere, tropics, and Southern Hemisphere). Therefore, the best that can be done is to tune the parameterization so that the global (or hemispheric) average predicted innovation standard deviation matches the actual. The fact that the vertical structure does not match means that all of these system error parameterizations themselves are deficient, and do not correctly represent the vertical structure of the actual system error. In particular, the fact that the multiplicative inflation system error parameterization cannot match the actual vertical structure of the innovation variance suggests that the structure of the underlying system error covariance is quite different than the background-error covariance represented by the dynamical ensemble, since the multiplicative inflation parameterization can only represent the system error in the subspace of the existing ensemble. One can either add more tunable parameters to the parameterizations to force the structures to match, or try to develop new parameterizations that more accurately reflect the structure of the underlying system error covariance.

Equation (8) is derived by assuming that the background forecast and observation errors are uncorrelated, and the observations and background forecast are unbiased (i.e., the expected value of the mean of the innovation $\mathbf{y}^{o}-\mathbf{H} \overline{\mathbf{x}}^{b}$ is zero). Figure 9 shows the innovation bias with respect to radiosondes for the EDAaddinf and NCEP-Benchmark experiments [the other EDA experiments (not shown) have similar innovation biases]. There are significant temperature biases in the lower troposphere, most likely due to systematic errors in the forecast model's boundary layer parameterization. The temperature bias in the lower troposphere is a significant fraction of the root-mean-square fit of the background forecast to the radiosonde observations (Fig. 2). Meridional wind biases are also evident in the lower troposphere and near the tropopause, but they are much smaller relative to the root-mean-square fit. For the temperature field at least, the fact that the ensemble spread appears deficient in the lower troposphere can be partially explained by the bias component of the innovations, which is not accounted for in
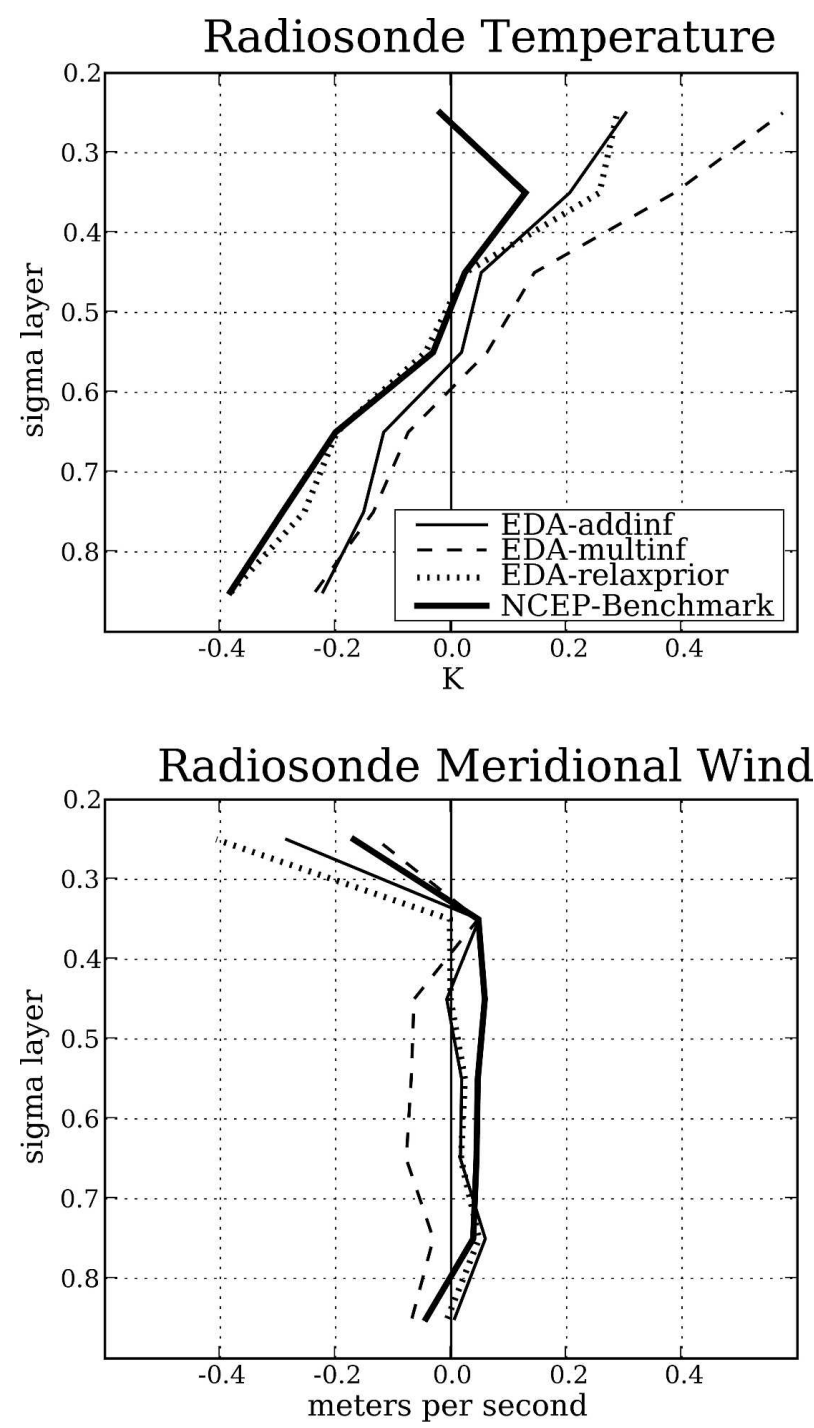

FIG. 9. Mean difference between 6-h forecast and radiosonde observations (bias) for forecasts initialized from 0600 and 1800 UTC EDA from 8 Jan to 8 Feb 2004. Three different EDA ensembles are shown; each employing a different method for parameterizing system error (see text for details).

(8). However, the mismatch between the predicted and actual meridional wind innovation standard deviation appears to primarily be a result of deficiencies in the parameterization of the system error covariance.

\section{d. Comparison with the LETKF}

The LETKF proposed by HKS is algorithmically very similar to the implementation used here, except that each state vector element is updated using all of the observations in the local region simultaneously using the Kalman filter update equations expressed in the subspace of the ensemble. We have performed an ex- 
N. Hem. Geopotential Height

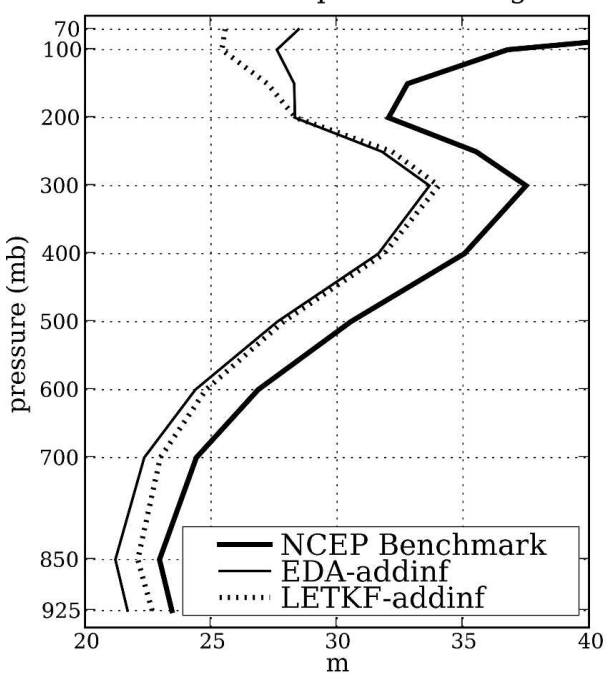

N. Hem. Meridional Wind

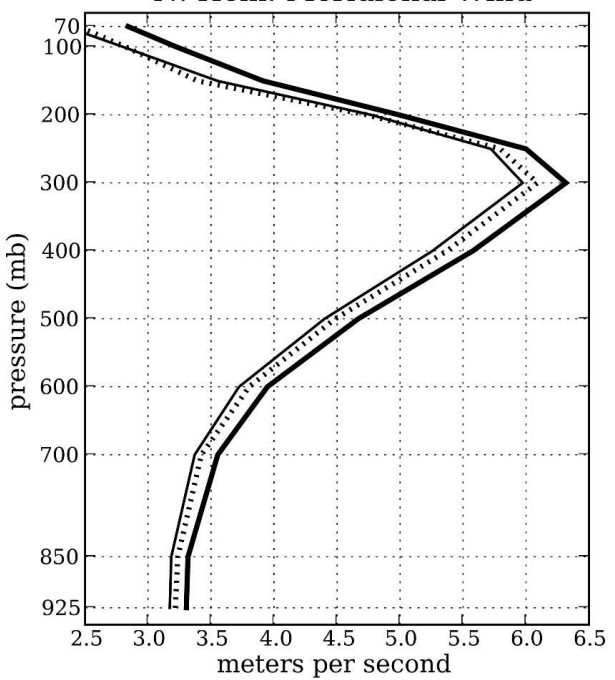

S. Hem. Geopotential Height

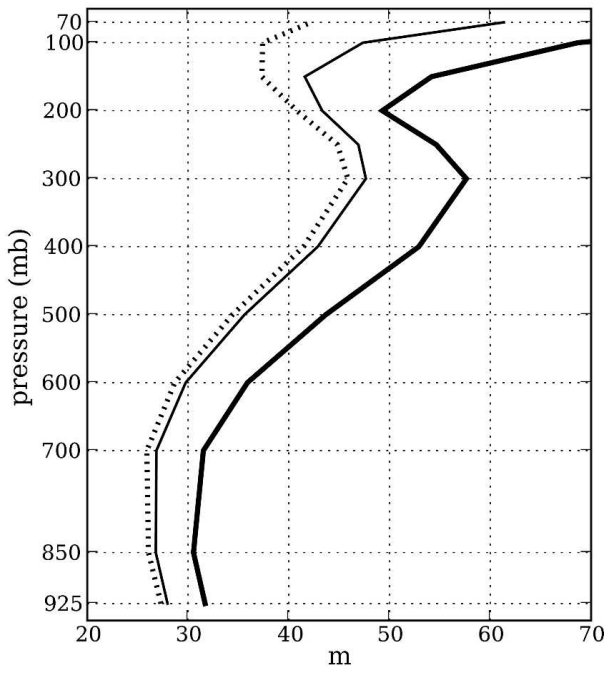

S. Hem. Meridional Wind

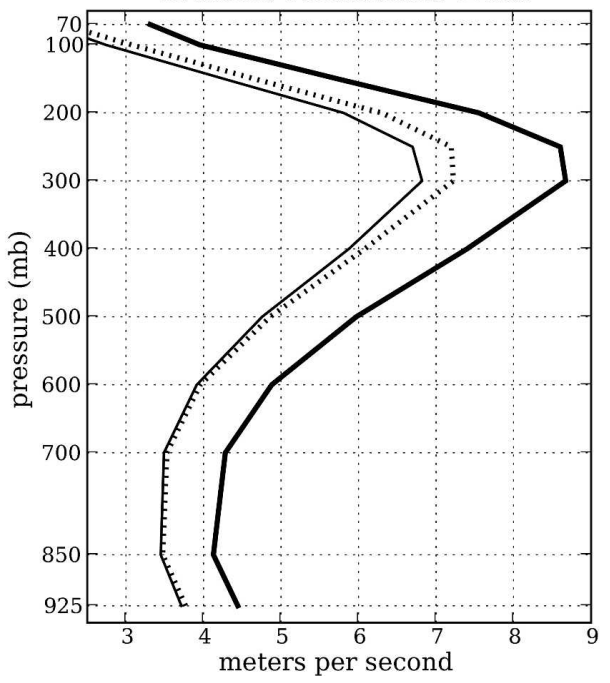

FIG. 10. Vertical profiles of 48-h geopotential height and meridional wind forecast error (measured relative to the NCEP-Operational analysis). Values for the Northern Hemisphere (poleward of $20^{\circ} \mathrm{N}$ ) and the Southern Hemisphere (poleward of $20^{\circ} \mathrm{S}$ ) are shown for the EDA-addinf, LETKF-addinf, and the NCEP-Benchmark experiments.

periment with LETKF with observation error localization, using additive inflation as a parameterization of system error (LETKF-addinf). The parameter settings for the experiment are identical to those used in the EDA-addinf experiment discussed previously. Figure 10 compares the 48 -h geopotential height and meridional wind forecast errors for forecasts initialized from the EDA-addinf, LETKF-addinf, and NCEPBenchmark analyses. The skill of the LETKF-addinf forecasts and EDA-addinf forecasts are very similar. The small differences between the LETKF-addinf and EDA-addinf experiments could be due to several factors. First, the method used for localizing the impact of observations is slightly different, as the LETKF localizes the impact of observations by increasing the observation error with distance away from the analysis point, while our serial processing implementation localizes the background-error covariances directly. Further experimentation is needed to see if these approaches are indeed equivalent in practice. Second, no adaptive observation thinning was done in the LETKF experiment. The fact that the quality of the EDA-addinf and LETKF-addinf forecasts are so similar suggests that the adaptive observation thinning did not have much of an impact, other than reducing the computational cost of the serial processing algorithm. Regarding computa- 
tional cost, we note that with the adaptive thinning algorithm, the cost of the two algorithms are similar. If the adaptive thinning is turned off, the serial processing algorithm is nearly an order of magnitude more expensive than the LETKF. Last, we may not have tuned the parameters to get the best performance from either the LETKF or the serial filter, and it is likely that the optimal parameters are not the same. The differences between the LETKF-addinf and EDA-addinf experiments are so small that we believe differences in tuning, rather than fundamental differences in the algorithms, are more likely the primary factor.

\section{Summary and discussion}

We have shown that ensemble data assimilation outperforms the NCEP 3DVAR system, when satellite radiances are withheld and the forecast model is run at reduced resolution (compared to NCEP operations). As expected from previous studies, the biggest improvement is in data-sparse regions. Since no satellite radiances were assimilated, the Southern Hemisphere is indeed quite data sparse. The background-error covariances in the NCEP 3DVAR system were retuned in the Southern Hemisphere to account for the lack of satellite radiances. The EDA analyses yielded a 24-h improvement in geopotential height forecast skill in the Southern Hemisphere extratropics relative to the reduced-resolution NCEP 3DVAR system, so that 48-h EDA-based forecasts are as accurate as 24-h 3DVARbased forecasts. Improvements in the data-rich Northern Hemisphere, while still statistically significant, were more modest (equivalent to a 6-h improvement in geopotential height forecast skill). For column-integrated water vapor, the EDA-based forecasts yielded a 12-h improvement in forecast skill in both the Northern and Southern Hemisphere extratropics. The fact that the improvement seen in the Northern Hemisphere is larger for the moisture field than the temperature field is consistent with the fact that in situ measurements of humidity are sparse in both hemispheres. It remains to be seen whether the magnitude of the improvements seen will be retained when satellite radiances are assimilated.

Three different parameterizations of system error (which is most likely dominated by model error) were tested. All three performed similarly, but a parameterization based on additive inflation using random samples of reanalysis 6-h differences performed slightly better in our tests. All of the parameterizations tested failed to accurately predict the structure of the forecast innovation variance, suggesting that further improvements in ensemble data assimilation may be achieved when methods for better accounting for the covariance structure of system error are developed. Significant innovation biases were found, primarily for lowertropospheric temperature, suggesting that bias removal algorithms for EDA [such as those proposed by Baek et al. (2006) and Keppenne et al. (2005)] could also significantly improve the performance of EDA systems.

We believe that these results warrant accelerated development of ensemble data assimilation systems for operational weather prediction. The limiting factor in the performance of these systems is almost certainly the parameterization of system error. Even without further improvements in the parameterization of model error, ensemble data assimilation systems should become more and more accurate relative to 3DVAR systems as forecast models improve and the amplitude of the model-error part of the background-error covariance decreases. Current-generation ensemble data assimilation systems are computationally competitive with their primary alternative, 4DVAR. However, they are considerably simpler to code and maintain, since an adjoint of the forecast model is not needed.

Acknowledgments. Fruitful discussions with C. Bishop, E. Kalnay, J. Anderson, I. Szyunogh, P. Houtekamer, F. Zhang, G. Compo, H. Mitchell, and B. Hunt are gratefully acknowledged, as are the comments of three anonymous reviewers. This project would not have been possible without the support of the NOAA THORPEX program, which partially funded this research through a grant, and NOAA's Forecast Systems Laboratory, which provided access to their High Performance Computing System. TMH was partially supported through National Science Foundation Grants ATM-0130154 and ATM-020561.

\section{APPENDIX}

\section{Retuning the NCEP GDAS Background Error}

The background error covariances in the NCEP GDAS were originally obtained using the so-called National Meteorological Center (NMC, now operating as NCEP) method (Parrish and Derber 1992). This method assumes the statistics of background error can be estimated from the covariances of differences between 48- and 24-h forecasts verifying at the same time. The background-error covariances used in the operational GDAS were obtained using T254L64 forecasts, initialized from an analysis that included satellite radiances. To retune the system for T62L28 resolution, we reran the NMC method using the difference between 48- and 24-h T62L28 forecasts, initialized from the op- 

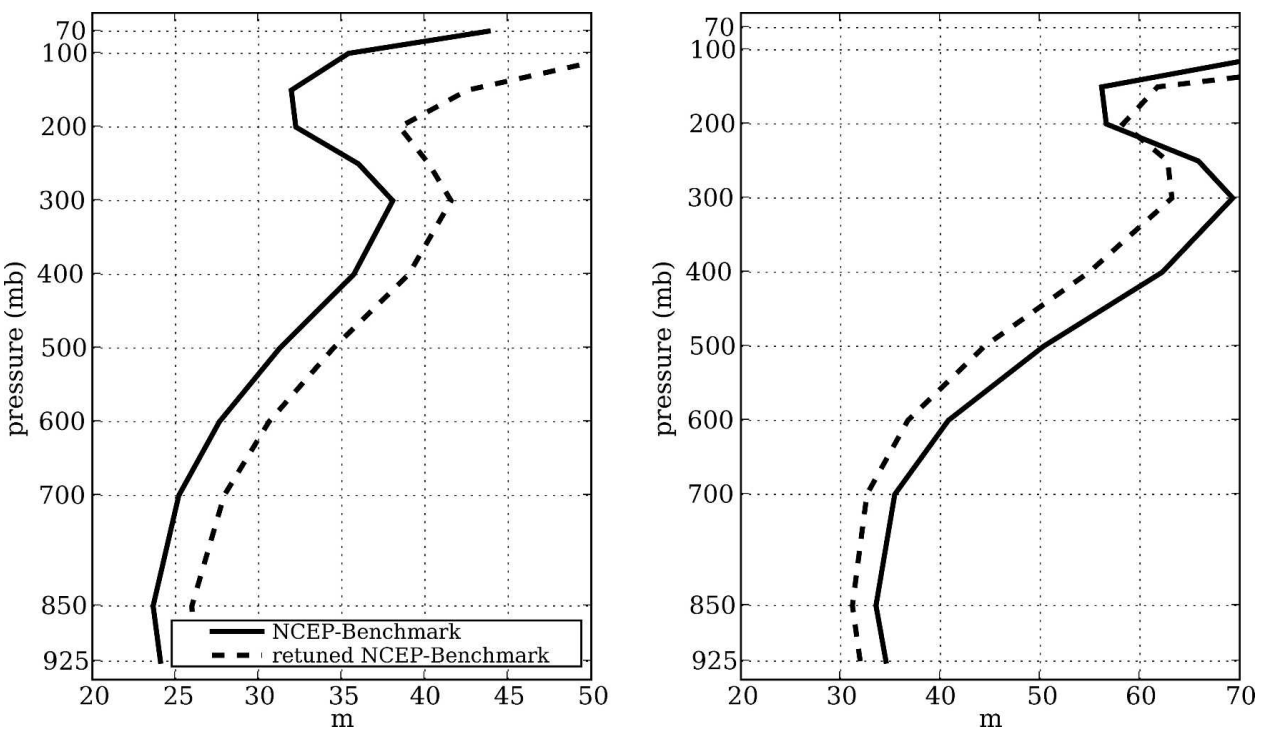

FIG. A1. Vertical profiles of 48-h forecast error, measured relative to the NCEP-Operational analysis, in the (left) Northern and (right) Southern Hemisphere extratropics (poleward of $20^{\circ}$ ). Forecasts were initialized four times daily between 0000 UTC 8 Jan and 0000 UTC 8 Feb 2004 from analyses that did not include satellite radiances. The solid line is for T62L28 forecasts initialized from a T62L28 run of the NCEP GDAS system, but using the operational background-error covariances (which were tuned for a T256L64 forecast model and an observing network that includes satellite radiances). The dashed line is for T62L28 forecasts initialized from a run of the NCEP GDAS at T62L28 resolution, using backgrounderror covariances determined from the difference between 48- and 24-h T62L28 forecasts via the NMC method.

erational analyses for January and February 2004. Figure A1 shows the 48-h forecast skill (relative to the operational analysis) for forecasts initialized from an NCEP GDAS T62L28 assimilation without satellite radiances. The solid curve denotes the skill of forecasts initialized from analyses generated with the operational background-error statistics, while the dashed curve denotes the skill of forecasts initialized from analyses generated with the new background-error statistics. Retuning the background-error statistics improves the forecasts in the Southern Hemisphere, but degrades the forecasts in the Northern Hemisphere. Further iterations of the NMC method (using 48- - 24-h forecasts initialized from analyses produced by the previous iteration) do not improve the forecast skill.

Since our attempt to retune the background-error statistics using the NMC method did not uniformly improve the analyses, we tried an alternative approach. In the Spectral Statistical Interpolation (SSI) system used in the NCEP GDAS, the structure of the backgrounderror covariances is the same everywhere over the globe. However, the amplitude of the background-error variances may vary with latitude. If we assume that the dominant effect of removing the satellite radiances and reducing the resolution of the forecast model is to modify the amplitude of the background error in the
Southern Hemisphere, without changing the structure of the background-error covariance, then modifying the latitudinally varying background-error variances in the Southern Hemisphere should improve the quality of the analyses there, without degrading the analyses in the Northern Hemisphere. Following this logic, we multiply the operational background error variances by the function

$$
\begin{array}{cc}
1 & \phi \geq 0 \\
1+b \sin ^{2} \phi & \phi<0
\end{array}
$$

where $\phi$ is latitude and $b$ is an arbitrary constant factor that controls the amplitude in the Southern Hemisphere extratropics. By numerical experimentation (running the assimilation for 1 month, and examining the skill of 48 -h forecasts relative to the operational analysis), we have found that $b=-0.75$ produces the best results. ${ }^{\mathrm{A} 1}$ Figure A2 shows the skill of 48 -h geo-

A1 A negative value of the $b$ parameter implies that the background error variance is reduced in the Southern Hemisphere relative to the operational value. This is a somewhat surprising result, since we expected the background error variance would need to be increased for the no-sat observation network. Independent calculations performed at NCEP by one of the coauthors (Y. Song) have confirmed that analyses excluding satellite radiances are degraded when $b>0$. 

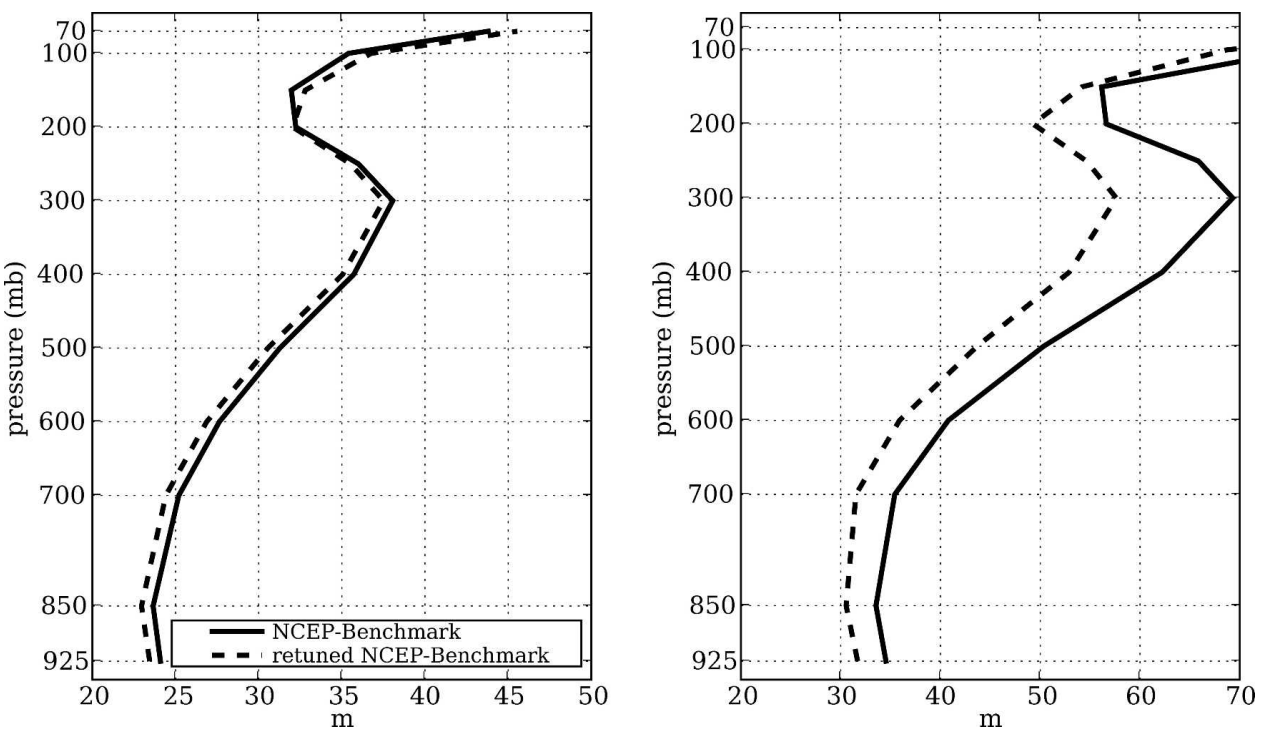

FIG. A2. As in Fig. A1 except that the thick dashed line represents T62L28 forecasts initialized from a T26L28 run of the NCEP GDAS system with the background-error variances modified only in the Southern Hemisphere, as described in the appendix.

potential height forecasts for the T62L28 "no-sat" experiments, using the operational (solid line) and retuned (dashed line) background error variances. The no-sat forecast error is reduced by $13 \%$ at $500 \mathrm{hPa}$ in the Southern Hemisphere, and the forecast skill in the Northern Hemisphere is changed little. We have used these analyses, which we refer to as the NCEPBenchmark analyses, throughout this study as a yardstick to measure EDA performance.

\section{REFERENCES}

Anderson, J. L., 2001: An ensemble adjustment Kalman filter for data assimilation. Mon. Wea. Rev., 129, 2884-2903.

—_, and S. L. Anderson, 1999: A Monte Carlo implementation of the nonlinear filtering problem to produce ensemble assimilations and forecasts. Mon. Wea. Rev., 127, 2741-2758.

Baek, S.-J., B. R. Hunt, E. Kalnay, E. Ott, and I. Szunyogh, 2006: Local ensemble Kalman filtering in the presence of model bias. Tellus, 58A, 293-306.

Bishop, C. H., B. Etherton, and S. J. Majumdar, 2001: Adaptive sampling with the ensemble transform Kalman filter. Part I: Theoretical aspects. Mon. Wea. Rev., 129, 420-436.

Bouttier, F., 1994: A dynamical estimation of forecast error covariances in an assimilation system. Mon. Wea. Rev., 122, 23762390.

Burgers, G., P. J. van Leeuwen, and G. Evensen, 1998: Analysis scheme in the ensemble Kalman filter. Mon. Wea. Rev., 126, 1719-1724.

Compo, G. P., J. S. Whitaker, and P. D. Sardeshmukh, 2006: Feasibility of a 100-year reanalysis using only surface pressure data. Bull. Amer. Meteor. Soc., 87, 175-190.

Courtier, P., J.-N. Thépaut, and A. Hollingsworth, 1994: A strategy for operational implementation of $4 \mathrm{D}$-Var, using an in- cremental approach. Quart. J. Roy. Meteor. Soc., 120, 13671387.

Daley, R., 1991: Atmospheric Data Analysis. Cambridge University Press, $457 \mathrm{pp}$.

Derber, J., and W.-S. Wu, 1998: The use of TOVS cloud-cleared radiances in the NCEP SSI analysis system. Mon. Wea. Rev., 126, 2287-2299.

Environmental Modeling Center, 2003: The GFS Atmospheric Model. NOAA/NCEP/Environmental Modeling Center Office Note 442, $14 \mathrm{pp}$. [Available online at http://www.emc. ncep.noaa.gov/officenotes/FullTOC.html.]

Evensen, G., 2003: The ensemble Kalman filter: Theoretical formulation and practical implementation. Ocean Dyn., 53, 343367.

Gaspari, G., and S. E. Cohn, 1999: Construction of correlation functions in two and three dimensions. Quart. J. Roy. Meteor. Soc., 125, 723-757.

Gelb, A., J. F. Kasper, R. A. Nash, C. F. Price, and A. A. Sutherland, 1974: Applied Optimal Estimation. The MIT Press, 374 pp.

Hamill, T. M., 2006: Ensemble-based data assimilation. Predictability of Weather and Climate, T. Palmer and R. Hagedorn, Eds., Cambridge Press, 124-156.

- , and C. Snyder, 2000: A hybrid ensemble Kalman filter-3D variational analysis scheme. Mon. Wea. Rev., 128, 2905-2919.

_ , and J. S. Whitaker, 2005: Accounting for the error due to unresolved scales in ensemble data assimilation: A comparison of different approaches. Mon. Wea. Rev., 133, 3132-3147.

,$- \ldots$, and C. Snyder, 2001: Distance-dependent filtering of background error covariance estimates in an ensemble Kalman filter. Mon. Wea. Rev., 129, 2776-2790.

Houtekamer, P. L., and H. L. Mitchell, 1998: Data assimilation using an ensemble Kalman filter technique. Mon. Wea. Rev., 126, 796-811.

—, and —, 2001: A sequential ensemble Kalman filter for atmospheric data assimilation. Mon. Wea. Rev., 129, 123-137. 
- and - 2005: Ensemble Kalman filtering. Quart. J. Roy. Meteor. Soc., 131, 3269-3289.

_ L. Lefaivre, J. Derome, H. Ritchie, and H. L. Mitchell, 1996: A system simulation approach to ensemble prediction. Mon. Wea. Rev., 124, 1225-1242.

_ , H. L. Mitchell, G. Pellerin, M. Buehner, M. Charron, L. Spacek, and B. Hansen, 2005: Atmospheric data assimilation with an ensemble Kalman filter: Results with real observations. Mon. Wea. Rev., 133, 604-620.

Hunt, B., E. Kostelich, and I. Syzunogh, 2007: Efficient data assimilation for spatiotemporal chaos: A local ensemble transform Kalman filter. Physica D, 230, 112-126.

Ide, K., P. Courtier, M. Ghil, and A. C. Lorenc, 1997: Unified notation for data assimilation: Operational, sequential, and variational. J. Meteor. Soc. Japan, 75B, 181-189.

Kalnay, E., L. Hong, T. Miyoshi, S.-C. Yang, and J. BallabreraPoy, 2007: 4D-Var or ensemble Kalman filter? Tellus, 59A, 758-773.

Kaminski, P. G., J. A. E. Bryson, and S. F. Schmidt, 1971: Discrete square-root filtering: A survey of current techniques. IEEE Trans. Automatic Control, 16, 727-736.

Keppenne, C. L., M. M. Rienecker, N. P. Kurkowski, and D. A. Adamec, 2005: Ensemble Kalman filter assimilation of temperature and altimeter data with bias correction and application to seasonal prediction. Nonlinear Processes Geophys., 12, 491-503.

Kistler, R., and Coauthors, 2001: The NCEP-NCAR 50-Year Reanalysis: Monthly means CD-ROM and documentation. Bull. Amer. Meteor. Soc., 82, 247-268.

Liu, Z.-Q., and F. Rabier, 2002: The interaction between model resolution, observation resolution and observation density in data assimilation: A one-dimensional study. Quart. J. Roy. Meteor. Soc., 128, 1367-1386.

Lorenc, A. C., 1986: Analysis methods for numerical weather prediction. Quart. J. Roy. Meteor. Soc., 112, 1177-1194.

Lynch, P., and X.-Y. Huang, 1992: Initialization of the HIRLAM model using a digital filter. Mon. Wea. Rev., 120, 1019-1034.

Meng, Z., and F. Zhang, 2007: Tests of an ensemble Kalman filter for mesoscale and regional-scale data assimilation. Part II: Imperfect model experiments. Mon. Wea. Rev., 135, 1403 1423.

Miyoshi, T., and S. Yamane, 2007: Local ensemble transform Kalman filtering with an AGCM at T159/L60 resolution. Mon. Wea. Rev., 135, 3841-3861.

NCEP Environmental Modeling Center, 2004: SSI Analysis System 2004. NOAA/NCEP/Environmental Modeling Center
Office Note 443, $11 \mathrm{pp}$. [Available online at http://www.emc. ncep.noaa.gov/officenotes/FullTOC.html.]

Ochotta, T., C. Gebhardt, D. Saupe, and W. Wergen, 2005: Adaptive thinning of atmospheric observations in data assimilation with vector quantization and filtering methods. Quart. J. Roy. Meteor. Soc., 131, 3427-3437.

Oppenheim, A., and R. W. Schafer, 1989: Discrete-Time Signal Processing. Prentice Hall, 879 pp.

Ott, E., and Coauthors, 2004: A local ensemble Kalman filter for atmospheric data assimilation. Tellus, 56A, 415-428.

Parrish, D., and J. Derber, 1992: The National Meteorological Center's spectral statistical-interpolation analysis system. Mon. Wea. Rev., 120, 1747-1763.

Pires, C., R. Vautard, and O. Talagrand, 1996: On extending the limits of variational assimilation in nonlinear chaotic systems. Tellus, 48A, 96-121.

Rabier, F., H. Järvinen, E. Klinker, J.-F. Mafouf, and A. Simmons, 2000: The ECMWF operational implementation of fourdimensional variational assimilation. I: Experimental results with simplified physics. Quart. J. Roy. Meteor. Soc., 126, 1143-1170

Simmons, A. J., and A. Hollingsworth, 2002: Some aspects of the improvement in skill of numerical weather prediction. Quart. J. Roy. Meteor. Soc., 128, 647-677.

Snyder, C., and F. Zhang, 2003: Assimilation of simulated Doppler radar observations with an ensemble Kalman filter. Mon. Wea. Rev., 131, 1663-1677.

Thépaut, J.-N., and P. Courtier, 1991: Four-dimensional data assimilation using the adjoint of a multilevel primitive equation model. Quart. J. Roy. Meteor. Soc., 117, 1225-1254.

Tippett, M. K., J. L. Anderson, C. H. Bishop, T. M. Hamill, and J. S. Whitaker, 2003: Ensemble square root filters. Mon. Wea. Rev., 131, 1485-1490.

Whitaker, J. S., and T. M. Hamill, 2002: Ensemble data assimilation without perturbed observations. Mon. Wea. Rev., 130, 1913-1924.

— G. P. Compo, and T. M. Hamill, 2004: Reanalysis without radiosondes using ensemble data assimilation. Mon. Wea. Rev., 132, 1190-1200.

Wilks, D. S., 2006: Statistical Methods in the Atmospheric Sciences. 2d ed. Academic Press, 467 pp.

Zhang, F., C. Snyder, and J. Sun, 2004: Impacts of initial estimate and observation availability on convective-scale data assimilation with an ensemble Kalman filter. Mon. Wea. Rev., 132, $1238-1253$ 